\title{
Impact of Tropical Storm Bopha on the Intensity Change of Super Typhoon Saomai in the 2006 Typhoon Season
}

\author{
Hongxiong Xu, ${ }^{1}$ Xuejin Zhang, ${ }^{2}$ and Xiangde $\mathrm{Xu}^{3}$ \\ ${ }^{1}$ School of Atmospheric Physics, Nanjing University of Information Science \& Technology Nanjing, Jiangsu 210044, China \\ ${ }^{2}$ University of Miami, Cooperative Institute for Marine and Atmospheric Studies, Miami, FL 33149, USA \\ ${ }^{3}$ State Key Laboratory of Severe Weather, Chinese Academy of Meteorological Sciences, Beijing 100081, China
}

Correspondence should be addressed to Xuejin Zhang; xuejin.zhang@noaa.gov

Received 2 March 2013; Accepted 7 May 2013

Academic Editor: Lian Xie

Copyright (C) 2013 Hongxiong Xu et al. This is an open access article distributed under the Creative Commons Attribution License, which permits unrestricted use, distribution, and reproduction in any medium, provided the original work is properly cited.

\begin{abstract}
Super Typhoon Saomai (2006, 08W), which caused historical disaster in the landfall region, is the most powerful typhoon ever making landfall in Mainland China since 1949. The impact of Tropical Storm Bopha $(2006,10 \mathrm{~W})$ on Saomai is regarded as a binary tropical cyclone (TC) interaction. In order to quantify the influence of Bopha on the intensity of Saomai, a set of numerical experiments are performed by artificially modifying the intensity of Bopha in its initial conditions. It is shown that changing the intensity of Bopha has significant effects on simulating Saomai's intensities, structures, and tracks. We find that moisture transport is a pivotal process of binary TC interaction. It is interesting that there are opposite effects by Bopha at different development stages of Saomai. The existence of Bopha and increasing its intensity would weaken Saomai at its intensifying stage while intensifying Saomai at its weakening stage. A possible explanation of these effects is the direction change of moisture transport from/to Saomai at its intensifying/weakening stages through the channel. It may suggest a significant relevance for operational intensity forecasts under active binary TC interaction.
\end{abstract}

\section{Introduction}

Tropical cyclones (TCs), especially super typhoons (the terms "hurricane" and "typhoon" are regionally specific names for a strong tropical cyclone. Typhoon is the name of tropical cyclone in the northwest Pacific Ocean west of the dateline. Super typhoon is the strongest category in the typhoon classification (C. Landsea, http://www.aoml.noaa.gov/hrd/tcfaq/ A1.html)), are one of the most powerful natural phenomena, which may bring torrential precipitation, dangerous storm surge, flash flooding, and disastrous winds to the impacted areas. Thus, it is of great interests to accurately predict tracks, intensities, and the abrupt intensity changes of TCs in operational forecasts. Apparently, TC intensity change interweaves multiscale nonlinear interactions among different physical processes and weather systems $[1,2]$. Such interactions include environmental moisture transport and binary TC (BTC) interaction.
As early as the 1920s, scientists began to note interaction phenomena between two vortexes in the tropics such as BTCs in the tropics, that is, binary vortices phenomenon or the Fujiwhara effect [3-5]. Later, Brand [6] studied 22 cases of BTCs over the North Pacific. The study found that BTC interaction could occur when the distance of two TC centers is less than 700 nautical miles. It also showed that the effect of such a BTC interaction depends on sizes, structures, intensities, and the ambient circulations of TCs. Concerning the more complicated and realistic flow patterns surrounding tropical cyclones, studies by Carr et al. and Carr and Elsberry $[7,8]$ detailed three conceptual models to categorize the BTC interaction processes and objective identification criteria of the interaction categories.

The observational and theoretical aspects of tropical cyclone interaction processes and their impact on TC's intensity were further investigated in a series of papers by Chen and Meng [9], Chen et al. [10], Wei and Zhang [11], and 
Kuo et al. [12]. Chen and Meng [9] and Chen et al. [10] pointed out that two TCs usually rotate counterclockwise around the centroid point on the connection line between two centers, attract each other, and tend to merge into one circulation system when the distance between two TCs is close enough. Wei and Zhang [11] conducted laboratory simulations and theoretical analyses of BTCs, which are similar to actual TCs dynamically and thermodynamically, to investigate their interaction. The results show that the rapid exchange of mass and momentum between two TCs is a primary physical process for their mutual rotation and attraction and evolution in their structures. Kuo et al. [12] suggests a correlation between the development and mutual interaction between Tropical Storm Alex $(1998,19 W)$ and Super Typhoon Zeb $(1998,18 \mathrm{~W})$. The cyclonic rotation of Alex around Zeb accelerated as the separation distance decreased to $850 \mathrm{~km}$. In the final stages of the interaction, Alex was quickly elongated and wrapped cyclonically around Zeb and thus became a spiral band of Zeb. It appeared that minimum Mean Sea Level Pressure (MSLP) of Zeb deepened from 960 to $920 \mathrm{hPa}$ within $24 \mathrm{~h}$ although the spiral band, which was originated by Alex, may never have mixed into the convective core of Zeb.

Many of the previous processes have also been examined by numerical studies of Yang et al. [13], Xu et al. [14], and Wu et al. [15]. Yang et al. [13] indicated that prediction of binary interaction is sensitive to the representation of initial vortex by studying the binary interaction between Typhoon Fengshen (2002) and Fungwong (2002). They further implied that the interaction is influenced by the monsoon trough and subtropical high as well. Xu et al. [14] found that the presence of Tropical Storm Goni $(2009,08 \mathrm{~W})$ at the upstream of Typhoon Morakot $(2009,09 \mathrm{~W})$ may be an important factor for the maintenance and intensification of Morakot. Goni transported a large amount of moisture and energy into Morakot. Numerical simulations indicate that the interaction between Goni and Morakot accounts for about 30\% more rainfall than no interaction when Goni did not present. However, Wu et al. [15] explained the relative effects between Morakot and Goni. At upper level, the northeasterly jet flow in the southwest quadrant of Morakot circulation enhanced the upper-level divergence of Goni and thus sustained and enhanced intensity of Goni. Meantime, Morakot, which possesses strong convergence and ascending airflow at the low level, supplies a stable southwesterly warm and wet flow through the transport channel, thus supporting the lowerlevel water vapor convergence of Goni. In contrast, Goni, which locates at the upstream of the southwesterly flow to maintain Morakot moisture convergence, intercepted part of the water vapor transportation and weakened the moisture convergence to Morakot. Their simulation results indicated that BTC interaction processes strongly affected the forecast of TC's intensity.

In this study, we discussed the interaction processes between Super Typhoons Saomai and Typhoon Bopha in August 2006. Specifically, we explored impacts of BTC interaction on modulating the intensity and structure of Saomai. In Section 2, we described the HWRF model configuration and design of numerical experiments used in this study. We presented the results of numerical simulations and detailed effects on Saomai intensity through BTC interaction in Section 3. Finally we summarize conclusions in Section 4.

\section{Model Description and Experiment Design}

The Hurricane Weather Research and Forecast System (HWRF) Model [16, 17], an advanced hurricane prediction system based on Weather Research and Forecast software framework [18], was developed at National Centers for Environmental Prediction's (NCEP's) Environmental Modeling Center (EMC) for understanding the hurricane forecast issues and improvement of hurricane prediction. The HWRF modeling system, based on the Non-Hydrostatic Mesoscale Model dynamic core $[19,20]$, is a high-resolution coupled airsea-land prediction model with a movable nested grid capable of following the moving TC [16]. The configuration of the HWRF system consists of outermost (parent) domain and the movable nested grid with resolutions of 27 and $9 \mathrm{~km}$ respectively and 42 vertical levels. This HWRF modeling system has been used for real-time operational forecasts at NCEP since 2007 and was made available to research community in April 2010. The rotated latitude-longitude projection is utilized to maximize efficiency. The discretization grid is based on the Arakawa-E grid. The parameterization schemes are briefly described in $[16,17,21]$.

HWRF uses a TC initialization and relocation algorithm to initialize the TC based on the observed position and intensity. The Grid Point Statistical Interpolation (GSI), a 3Dimensional Variational (3DVAR) Data Assimilation (DA) system, is implemented for initializing the environmental circulation outside the TC vortex area. The vortex initialization, relocation, and GSI DA produce the initial conditions to better match the actual TC observations. Therefore, a composite synthetic vortex, which is generated from bogus vortex or cycled from the vortex of the previous cycle, is assimilated in the observed TC location. The initialization of the HWRF model consists of four major steps: (1) create first guess fields from the Global Forecast System (GFS) analysis; (2) remove the TC vortex from the global analysis; (3) add the synthetic vortex through the vortex initialization procedure; and (4) assimilate any additional available observational data outside TC core, which the GFS has not assimilated yet by its analysis time then. Steps (3) and (4) provide major advantages over the conventional GFDL TC initialization procedure.

A series of sensitivity experiments are conducted to explore the impact on the intensity of Saomai and its relation with the BTC interaction. The NCEP Global Data Assimilation System (GDAS) (refer to http://www.emc .ncep.noaa.gov/modelinfo/index.html for GFS and GDAS documents) Final Operational Global Analyses $\left(1^{\circ} \times 1^{\circ}\right)$ are used as initial and boundary conditions. The CNTR simulation is integrated for $90 \mathrm{~h}$, starting from 0000 UTC 8 August, 2006 with two synthetic vortices generated from the vortex initialization procedure aforementioned. Without the initialization, the initial analyses of the Mean Sea Level Pressures (MSLPs) of Bopha and Saomai are $1003 \mathrm{hPa}$ and 
$996 \mathrm{hPa}$, respectively, which are much weaker than the besttrack MSLP analyses of $991 \mathrm{hPa}$ and $967 \mathrm{hPa}$ from the Joint Typhoon Warning Center (JTWC). After implanting the vortices of the two storms, the corresponded intensities reach $993 \mathrm{hPa}$ and $972 \mathrm{hPa}$, respectively, which are in close agreement with the best track analyses by JTWC.

Table 1 summarized the designed experiments in this study. In CNTR experiment, HWRF TC initialization and relocation algorithm is used to initialize the TC based on the JTWC position and intensity (MSLP of Saomai and Bopha are $967 \mathrm{hPa}$ and $991 \mathrm{hPa}$, resp.). In Bopha_rm experiment, the Bopha vortex was filtered from the first guess fields through a selective band filter [22]. In Bopha_wk experiment, the relocation algorithm was not applied because GFS had applied the same relocation procedure on its analysis and the TC intensity adopted intensity of the GFS analysis, where MSLP of Bopha is about $12 \mathrm{hPa}$ higher than the one in CNTR. In Bopha_str experiment, MSLP of Bopha was reduced to $967 \mathrm{hPa}$ through HWRF vortex initialization and relocation procedure.

\section{Results}

3.1. Synoptic Background. Super typhoon Saomai was the most powerful typhoon ever to make landfall on Mainland China since 1949, with an MSLP of $915 \mathrm{hPa}$ and a sustained maximum $10 \mathrm{~m}$ wind speed (VMAX) (refer to http://www.aoml.noaa.gov/hrd/tcfaq/D4.html for definition of maximum sustained surface wind by Christopher Landsea) of over $72 \mathrm{~m} \mathrm{~s}^{-1}$, as well as the record-breaking deepening rate of $25 \mathrm{hPa}$ in 18 hours. Saomai began at 0000 UTC 4 August, 2006, as a tropical depression (tropical depression (TD): $10.8-17.1 \mathrm{~m} \mathrm{~s}^{-1}$; tropical storm (TS): $17.2-24.4 \mathrm{~m} \mathrm{~s}^{-1}$; typhoon (TY): 32.7-41.4 $\mathrm{m} \mathrm{s}^{-1}$; super typhoon (STY): $\geq 51.0 \mathrm{~m} \mathrm{~s}^{-1}$ ) over the northwest Pacific Ocean. It intensified into a tropical storm at 0600 UTC 5 August and typhoon at 1200 UTC 6 August. Then it rapidly strengthened into super typhoon by 1200 UTC 9 August. Saomai made landfall on the coast of Zhejiang province $\left(27.20^{\circ} \mathrm{N}, 119.90^{\circ} \mathrm{E}\right)$ at $1200 \mathrm{UTC} 10$ August then weakened and dissipated inland $\sim 1000 \mathrm{~km}$ west of the landfall point. Meanwhile, after its genesis to the east of the Taiwan Island at 0000 UTC 5 August, tropical storm Bopha moved west-northwestward at 0600 UTC 6 August under the influence of favorable environmental circulation such as warm sea surface temperature. Then Bopha moved southwestward and crossed the island of Taiwan. It wandered for nearly $60 \mathrm{~h}$ in the South China Sea interacting with Super Typhoon Saomai. The large-scale environment of experiment initial time at 000008 August, 2006, is shown in Figure 1. Two cyclonic circulations at the surface locate in row in the east of Taiwan Island. A strong subtropical high on the $500 \mathrm{hPa}$ stations in the east of Shikoku Island. The low center of Saomai is aligned with the surface circulation, which suggests that Saomai has become a mature typhoon. The low center of Bopha is tilted to the south on the upper level in the shear environment resulting from Saomai's outskirt flow in the upper level. Bopha is prevented further intensification in
TABLE 1: List of designed experiments.

\begin{tabular}{lcl}
\hline Experiment & Name & Remarks \\
\hline 1 & CNTR & $\begin{array}{l}\text { The initial MSLP used in the vortex } \\
\text { initialization procedure of Saomai } \\
\text { and Bopha is } 967 \mathrm{hPa} \text { and } 991 \mathrm{hPa}, \\
\text { respectively } \\
\text { As CNTR, but remove Bopha vortex } \\
2\end{array}$ \\
Bopha_rm & $\begin{array}{l}\text { As CNTR, but the vortex } \\
\text { initialization procedure is not used in } \\
\text { Bopha vortex. } \\
\text { As CNTR, but with MSLP of Bopha } \\
967 \mathrm{hPa}\end{array}$ \\
\hline
\end{tabular}

such an environment although a strong warm center of SST is just beneath it.

3.2. Numerical Experiment Analysis. Figure 2 presents the simulated track of CNTR, initiated at 0000 UTC 08 AUG, and the observed best tracks from the Joint Typhoon Warning Center (JTWC) and China Meteorological AdministrationShanghai Typhoon Institute (CMA-SH). CNTR demonstrates that the track of Bopha is in good agreement with the observed tracks in the first $30 \mathrm{hr}$ simulation, while it generally tends to a southward bias in the final $60 \mathrm{~h}$ simulation. Except for slight overestimation of the translation speed after $36 \mathrm{hrs}$, the simulated track of Saomai is nearly the same as the observed track from the JTWC and CMA-SH. The simulated intensities of Saomai and Bopha in CNTR and those from JTWC and CMA-SH are shown in Figure 3. Despite the differences in the detailed intensity, the model simulates reasonably well the initial intensification, the maintaining stage, and the subsequent weakening stage, respectively. The intensity change timing is almost identical to the observed. In short, the simulated track and intensity in CNTR show the reasonable track and intensity evolutions inspite of underprediction of the peak intensity of Saomai.

Meanwhile, there may exist BTC interactions between Saomai and Bopha as the two TCs approach each other within a certain range as indicated by other studies (e.g., [8-10]). We designed a group of sensitivity experiments to investigate the processes. First, the influence of different Bopha intensities on Saomai's track is examined by comparing experiments Bopha_rm, Bopha_wk, and Bopha_str with CNTR. Figure 4 displays the simulated tracks in these experiments from 0000 UTC 8 August to 0000 UTC 12 August. All of experiments show virtually identical tracks of Saomai in the initial $30 \mathrm{hr}$ simulation. The track of Saomai of the Bopha_str diverges towards the right of the CNTR in the final $60 \mathrm{hr}$ simulation. In contrast, the track of Saomai of the Bopha_wk deviates towards the left of the CNTR, and the track of Saomai of the Bopha_rm shifts further leftward and becomes almost westward track. These simulated track characteristics indicate that the existence of Bopha may play an important role on Saomai's track. The results further indicate that different intensity of Bopha may result in more impact on the intensity of Saomai than on its track as indicated in CNTR, Bopha_wk, and Bopha_str. As pointed out in Yang et al. [13], the BTC 


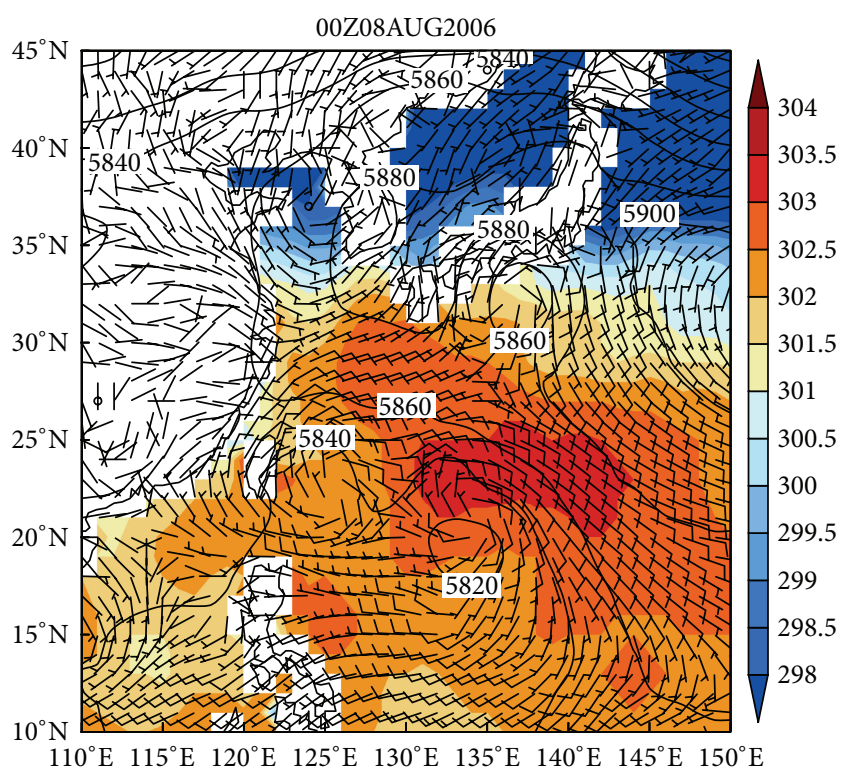

FIGURE 1: Geopotential height at $500 \mathrm{hPa}$ (contour, 20-meter interval), SST (shaded), and wind (vector) at 10 -meter wind from the $1^{\circ} \times 1^{\circ}$ NCEP FNL operational global analysis at 0000 UTC 8 August, 2006 .

interaction process is sensitive to initial conditions, especially in weaker TCs.

The intensity evolutions of the two TCs in all experiments are shown in Figure 5. Saomai's simulated intensity progressed differently at different stages among the different experiments. Several interesting sensitivity characteristics in distinctive stages are worthy of further discussion. During the intensification stage, Saomai attained similar minimum MSLPs and maximum surface wind speed among all experiments despite different paces. During the weakening stage, the existence of Bopha was able to alter significantly the timing of the rapid intensity weakening process of Saomai. In general, Saomai's maximum intensity seems less sensitive to Bopha's intensity. However, at the intensification stage, Saomai obtains slightly stronger intensities in Bopha_rm and Bopha_wk than in CNTR. On the other hand, intensity of Saomai is weaker in Bopha_str than in CNTR. By contrast, during weakened stage of Saomai starting at 0000 UTC 10 August, the weakening rate monotonically depends on the initial intensities of Bopha. The deeper the initial Bopha vortexes are, the stronger intensities of Saomai attained at the weakening stage will be. The MSLP difference between two extreme experiments, Bopha_rm versus Bopha_str, is about $25 \mathrm{hPa}$ at $1800 \mathrm{UTC} 10$ August. The results of these numerical experiments demonstrate that the initial intensities of Bopha have a distinctive effect on Saomai at different stages. Saomai is intensified (weakened) and deviated northward (southward) when Bopha's initial intensity weakens (deepens) in intensifying (weakening) stage.

In addition to Bopha's effect on Saomai's track and intensity, Bopha also triggers the Saomai's different vertical structure response at the weakening and intensifying stages.

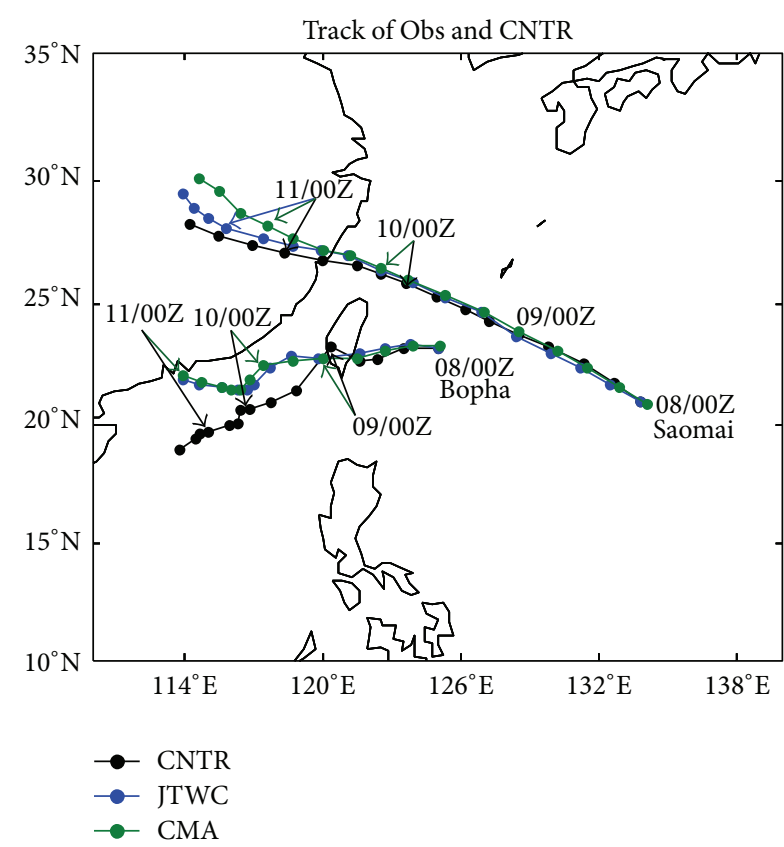

FIGURE 2: Best tracks of CMA and JTWC and simulated tracks of Bopha (2006, 09W) and Saomai (2006, 08W) from 0000 UTC 8 August to 1800 UTC 11 August, 2006.

Figure 6 shows the longitude-vertical sections of Saimai at the intensifying stage. CNTR (Figure 6(a)) also simulates the vertical extent of $50 \mathrm{~m} / \mathrm{s}$ region extending up to $450 \mathrm{hPa}$ on the east side as compared to $800 \mathrm{hPa}$ on the west side although the maximum winds on both sides are stronger than those in Bopha_rm and Bopha_wk. In Bopha_rm (Figure 6(b)), Saomai has nearly symmetric structure. Its narrow weak wind region $(<5 \mathrm{~m} / \mathrm{s})$ extends up to approximately $350 \mathrm{hPa}$ vertically and wind speed $>50 \mathrm{~m} / \mathrm{s}$ extend up to $380 \mathrm{hPa}$ on the east side of Saomai and $650 \mathrm{hPa}$ in the west side. In Bopha_wk (Figure 6(c)), the maximum wind speed $>50 \mathrm{~m} / \mathrm{s}$ extends up to about $550 \mathrm{hPa}$ on the eastside and $800 \mathrm{hPa}$ on west side. In Bopha_str, the weakening trend is further displayed (Figure 6(d)). These features suggest that Saomai becomes weaker and less symmetric with increasing intensity of Bopha at the intensifying stage.

The meridional vertical sections of Saomai's horizontal winds resulted from Bopha intensity sensitive experiments are shown the vertical structure at weakening stage (Figure 7). In contrast to the intensifying stage, Bopha's initial intensity on Saomai's intensity shows opposite effects at the weakening stage. The maximum winds reach $40 \mathrm{~m} / \mathrm{s}$ in Bopha_rm, $50 \mathrm{~m} / \mathrm{s}$ in Bopha_wk, and $55 \mathrm{~m} / \mathrm{s}$ in CNTR and Bopha_str, respectively. Conclusively, the stronger (weaker) the Bopha's initial intensities are, the weaker (stronger) Saomai's horizontal winds are resulted at the Saomai's intensifying stage. The opposite effects are shown at the Saomai's weakening stage. The vertical structure evolution of Saomai sensitive to Bopha's initial strength is also consistent with Saomai's intensity shown in Figure 5.

Figure 8(a) illustrates volume moisture fluxes computed from the NCEP FNL operational global analysis $1^{\circ} \times 1^{\circ}$ data 


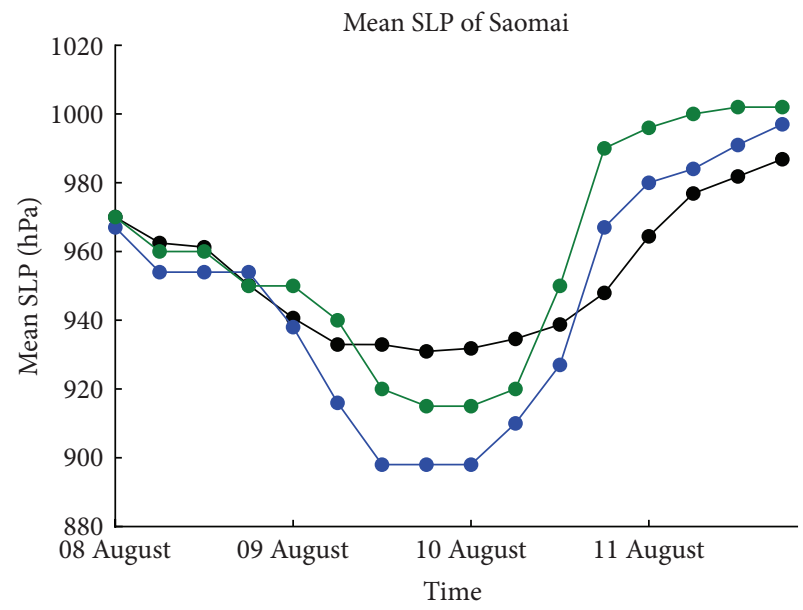

(a)
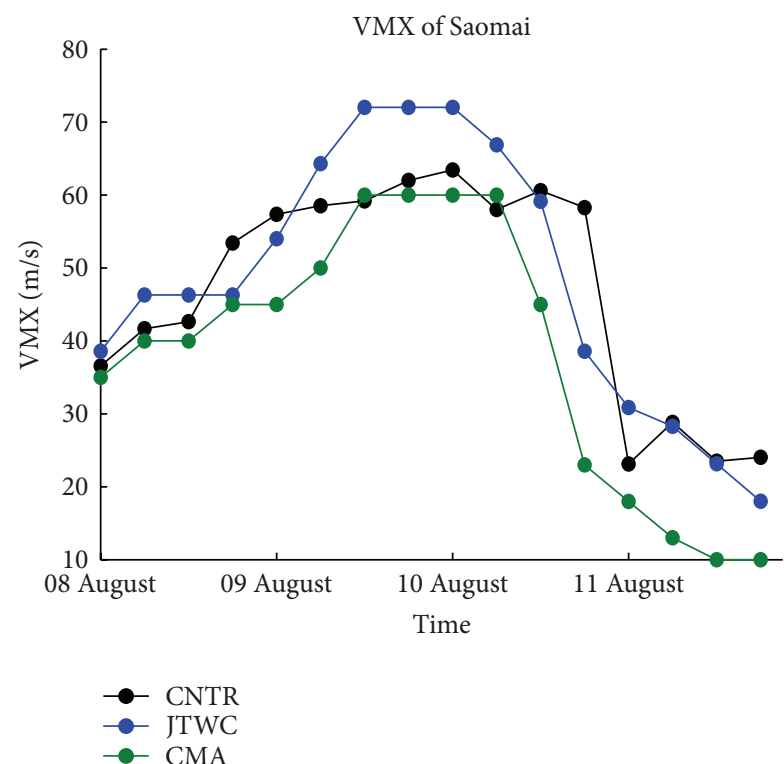

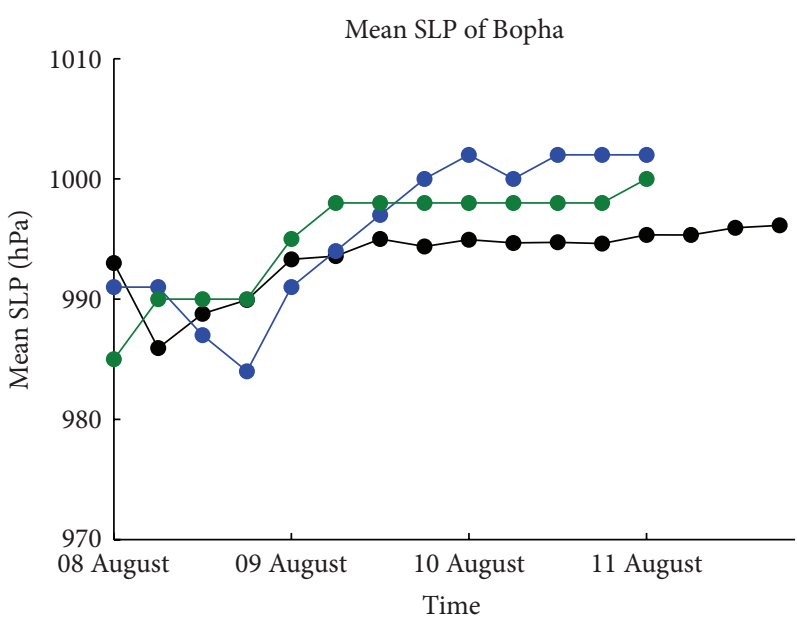

(b)

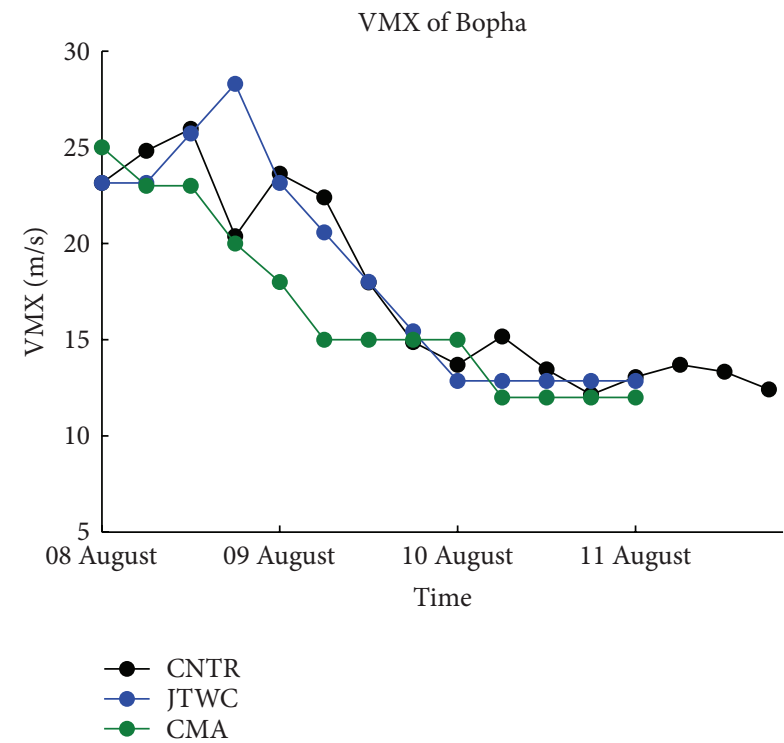

(d)

FIGURE 3: The 6-hourly minimum sea-level pressure (hPa) of best tracks of CMA and JTWC and simulated tracks of CNTR of Saomai (a) and Bopha (b) from 0000 UTC 8 August to 1800 UTC 11 August, 2006. (c) and (d) are corresponding to (a) and (b) except for 10-meter maximum wind.

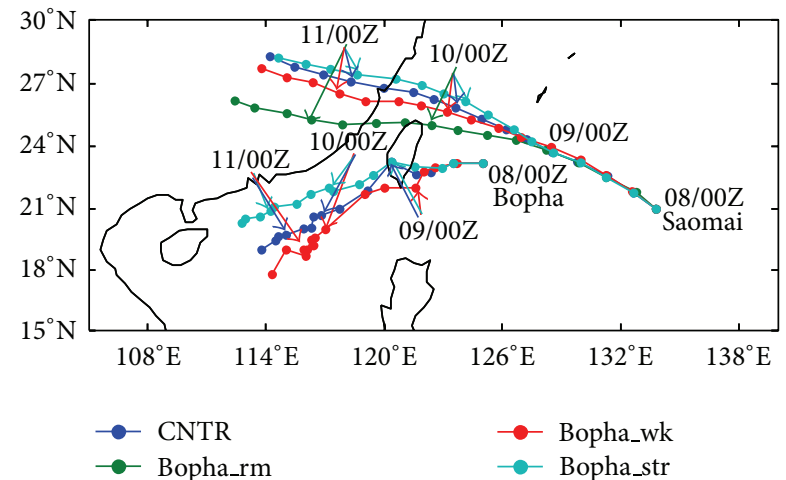

FIGURE 4: Simulated tracks of all sensitivity experiments at the 6hour interval from 0000 UTC 8 August to 1800 UTC 11 August, 2006. at 0600 UTC 09 August, 2006. We compute the volume moisture fluxes between surface and $100 \mathrm{hPa}$. The two components of the moisture flux are defined [14] as

$$
Q_{u}=\frac{1}{g} \int_{P_{t}}^{P_{s}} u q d p, \quad Q_{v}=\frac{1}{g} \int_{P_{t}}^{P_{s}} v q d p,
$$

where $P_{s}$ and $P_{t}$ are pressures at surface and $100 \mathrm{hPa}$, respectively, $q$ is specific humidity, $u$ and $v$ are horizontal wind components, and $g$ is the acceleration of gravity. There was a distinctive channel of moisture transport between Saomai and Bopha. The strong moisture fluxes display asymmetrical pattern around the connecting line between two TC centers. In contrast, the channel also exists between Saomai and Bopha at 0000 UTC 11 August, 2006 (Figure 8(b)), and 


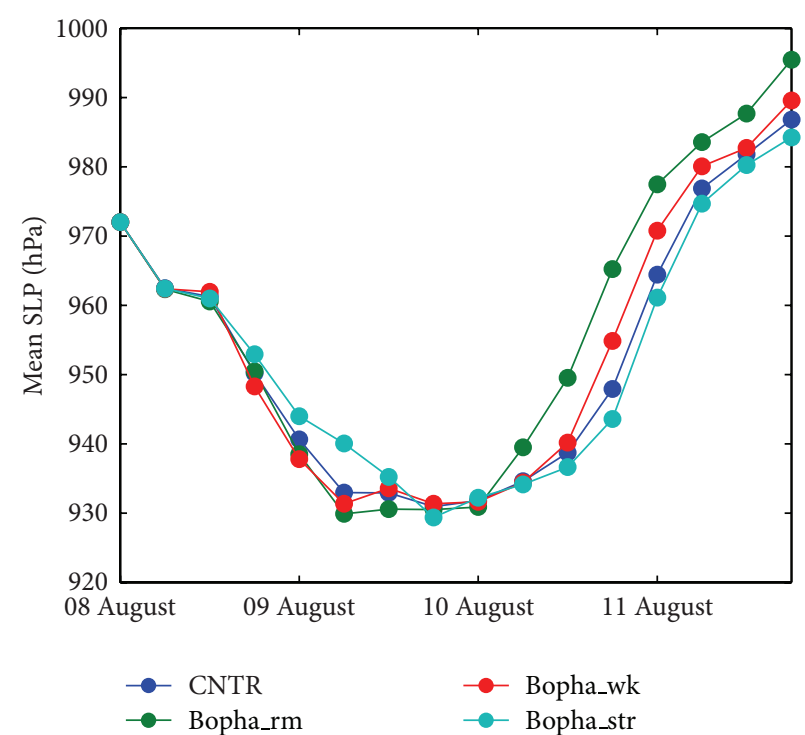

(a)

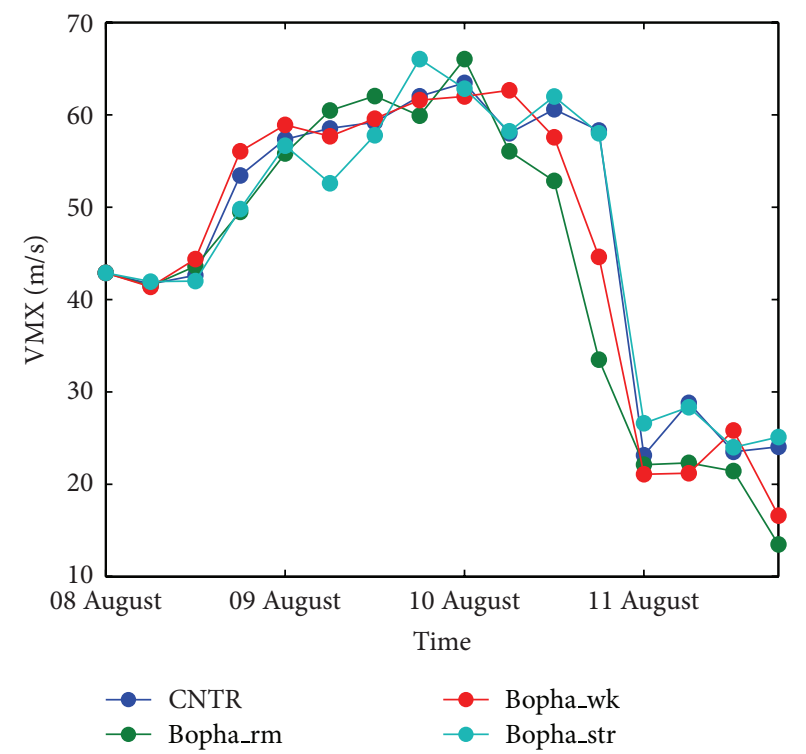

(c)

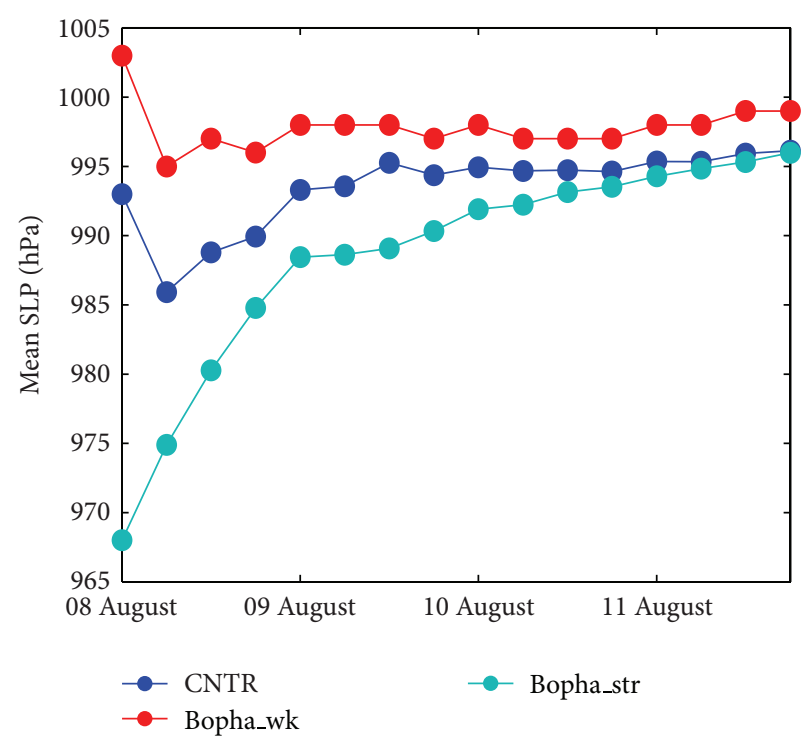

(b)

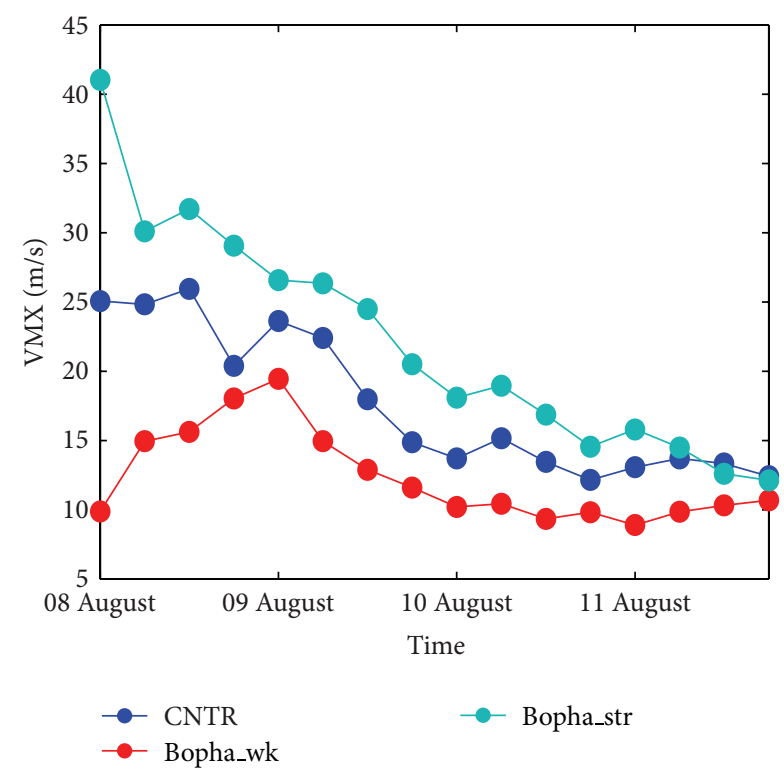

(d)

Figure 5: The 6-hourly minimum sea-level pressure (hPa) of Saomai (a) and Bopha (b) in each experiment from 0000 UTC 8 August to 1800 UTC 11 August, 2006. (c) and (d) are corresponding to (a) and (b) except for 10-meter maximum wind.

the rainbands located on the east side of the line (Figure omitted). Apparently, the rainbands are colocated within the channels, which are on the right side of the moisture transport direction along the line connecting two TC centers. These results indicate that the appearance of Bopha may be an important factor to modulate the Saomai's intensity. As pointed out in Xu et al. [14], BTCs interact with each other through the moisture and momentum transportations within the channels. Of interest is that the transportation direction within the channels shows opposite directions in different stages of Saomai's evolution because of the relative position of two TCs. Moisture is transported from Saomai to Bopha at the intensifying stage while the transport direction is reversed at the weakened stage. The direction change of moisture transport within the channels is resulted from the relative position between Saomai and Bopha and the moisture sources supply of moisture available to them. Noticeably, environmental moisture transported into Saomai exists directly from the southeast flow from the ocean. The moisture budget within Saomai can certainly influence the energy budget, resulting in the observed intensity fluctuation.

We also compare the volume moisture fluxes among the sensitivity experiments (Figures 9 and 10). Distribution of the moisture transport channel in CNTR agrees with Figure 8 except that the simulated magnitude is somewhat larger than NCEP FNL analysis. It is shown that the channel almost 


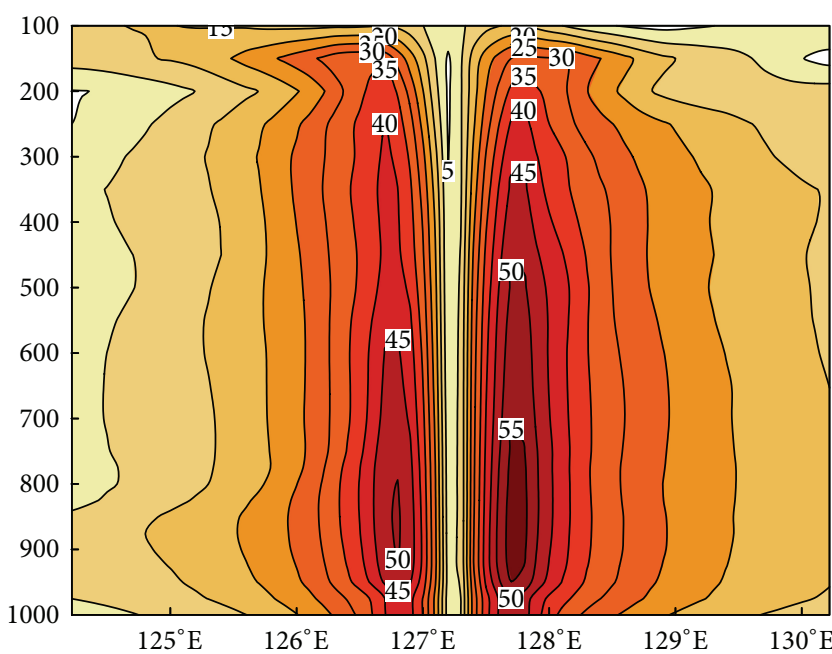

(a) $\operatorname{CNTR}\left(\right.$ Saomai at $127.2^{\circ} \mathrm{E}, 24.3^{\circ} \mathrm{N}$ )

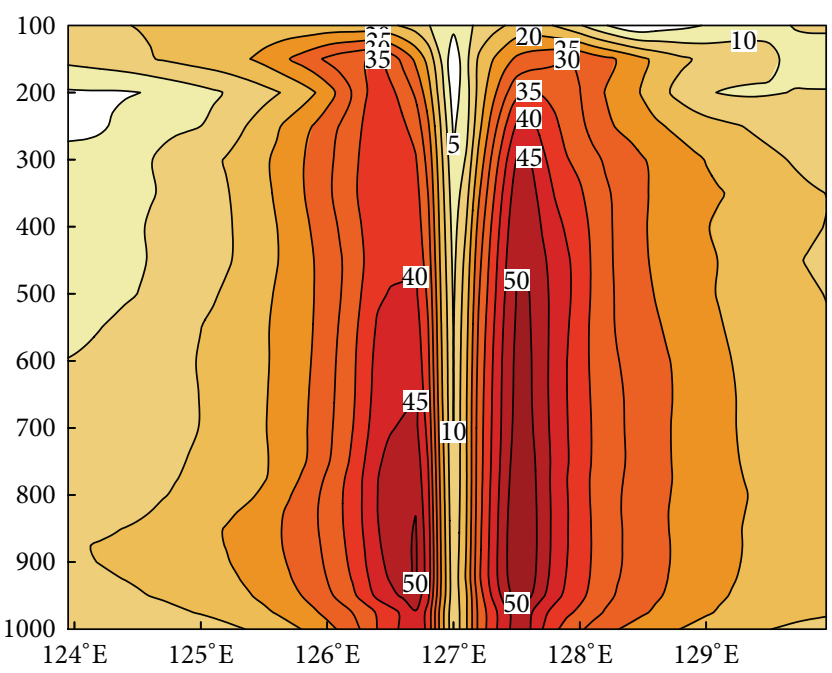

(c) Bopha_wk (Saomai at $127.0^{\circ} \mathrm{E}, 24.4^{\circ} \mathrm{N}$ )

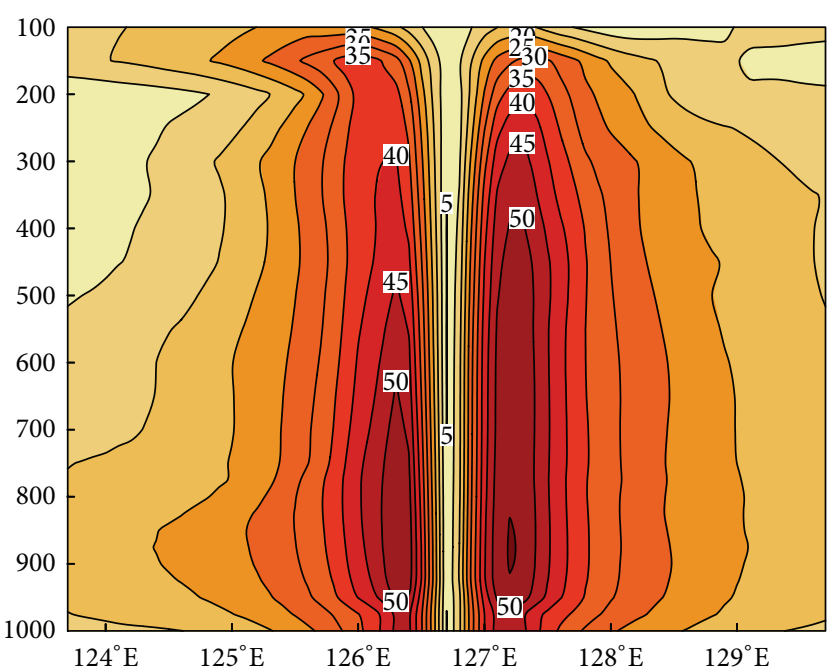

(b) Bopha_rm (Saomai at $126.0^{\circ} \mathrm{E}, 24.3^{\circ} \mathrm{N}$ )

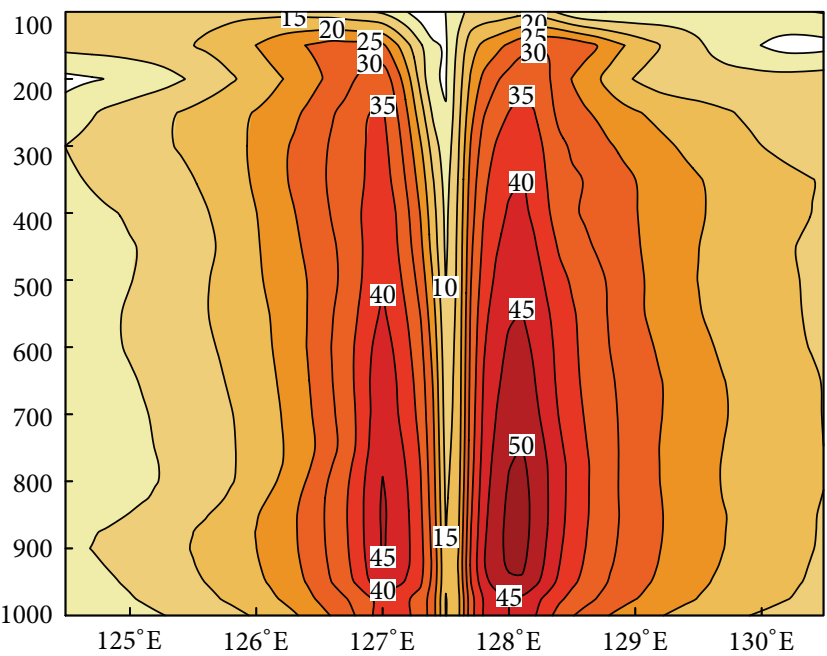

(d) Bopha_str $\left(\right.$ Saomai at $127.5^{\circ} \mathrm{E}, 24.2^{\circ} \mathrm{N}$ )

FIGURE 6: Horizontal wind of the meridional vertical cross-sections along the Saomai center $(\mathrm{m} / \mathrm{s}$, shaded) at the intensifying stage for CNTR (a), Bopha_rm (b), Bopha_wk (c), and Bopha_str (d) at 0600 UTC 09 August, 2006.

disappears in Bopha_rm. The difference between CNTR and Bopha_rm implies the important role of Bopha in the formation of the channel of moisture transport. In general, the sensitivity of the channels is consistent to Bopha's initial intensity and is reflected the distinction at different stages of Saomai's development. The peak tangential moisture fluxes in the channels increase from $700 \mathrm{~kg} \mathrm{~m}^{-1} \mathrm{~s}^{-1}$ in Bopha_wk, slightly bigger in CNTR, and $800 \mathrm{~kg} \mathrm{~m}^{-1} \mathrm{~s}^{-1}$ in Bopha_str (Figure 9) at the intensifying stage. In contrast, the peak tangential moisture fluxes in the channels are almost the same as those at the intensifying stage, while the strongest is in Bopha_str, followed in CNTR, and the weakest is in Bopha_rm (Figure 10) at the Saomai's weakening stage.

In order to corroborate the moisture transportation, FLEXPART, a Lagrangian particle dispersion model, was utilized to trace trajectories of water vapor particles based the NCEP Climate Forecast System Reanalysis (CFSR) 0.5-degree products [23]. A description of the model was documented in Stohl et al. [24]. FLEXPART calculates the trajectories of so-called tracer particles using the mean winds interpolated from the meteorological input fields plus random motions representing turbulence [25]. For moist convective transport, FLEXPART uses the scheme of Emanuel and ŽivkovićRothman [26], as described and tested by Forster et al. [27]. Figure 11 describes backward trajectories of the particle cluster released in the Bopha $\left(115.5 \sim 125.5^{\circ} \mathrm{E}, 20.1 \sim 25.0^{\circ} \mathrm{N}\right)$ at 1200 UTC 9 August, 2006. Initially, the particle cluster advances along the backward trajectories (Figure 11(a)). The cluster then appears to connect Saomai at the east side (Figure 11(b)). This connection is more obvious at 0600 UTC 8 August (Figure 11(c)). The cluster mixes into southeast flow (Figure 11(d)). Figure 12 shows the cluster trajectories released in Saomai $\left(114.5 \sim 120.5^{\circ} \mathrm{E}, 26 \sim 33.5^{\circ} \mathrm{N}\right)$ at $0000 \mathrm{UTC}$ 13 August, 2006. There are two branches of moisture transport 


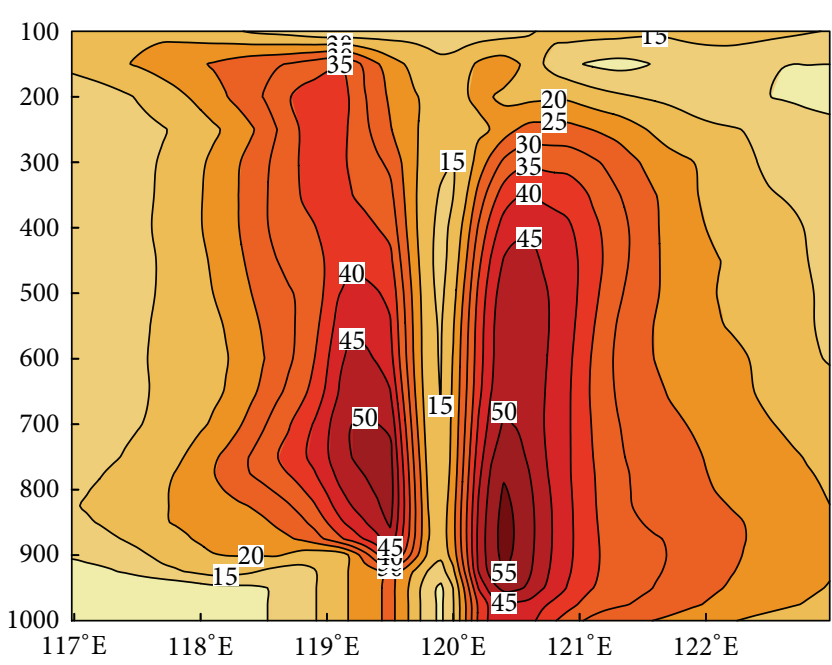

(a) $\operatorname{CNTR}\left(\right.$ Saomai at $\left.120.0^{\circ} \mathrm{E}, 26.8^{\circ} \mathrm{N}\right)$

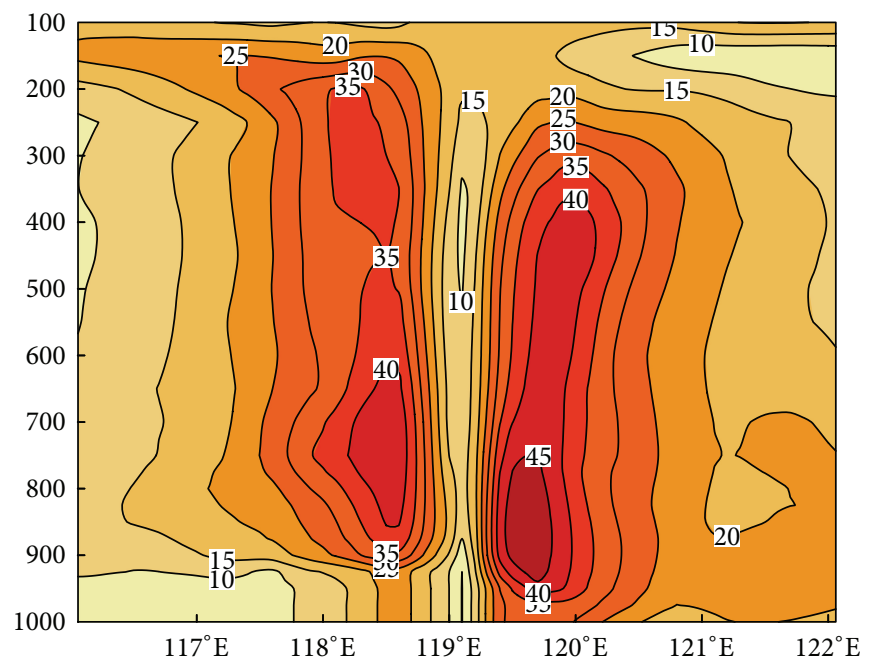

(c) Bopha_wk (Saomai at $119.1^{\circ} \mathrm{E}, 26.2^{\circ} \mathrm{N}$ )

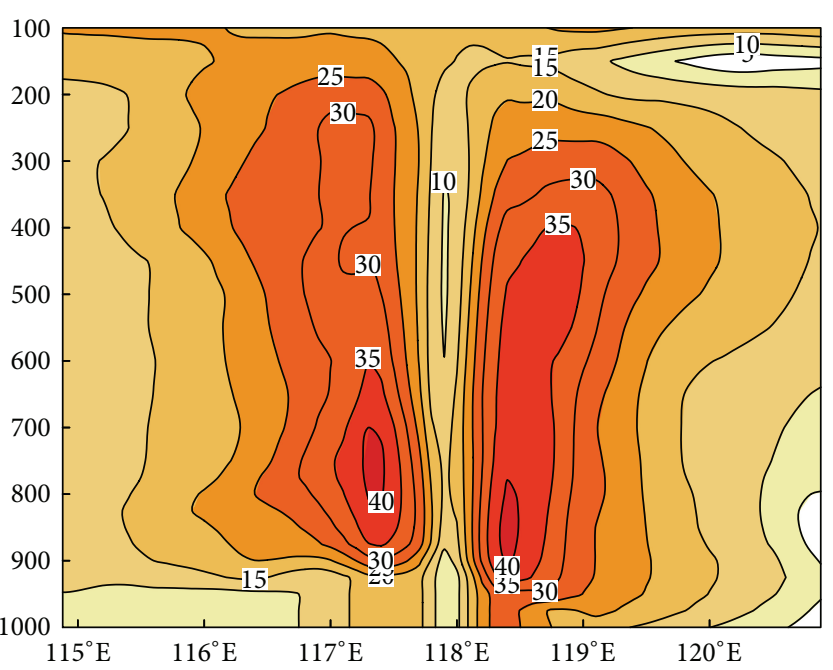

(b) Bopha_rm (Saomai at $117.9^{\circ} \mathrm{E}, 25.0^{\circ} \mathrm{N}$ )

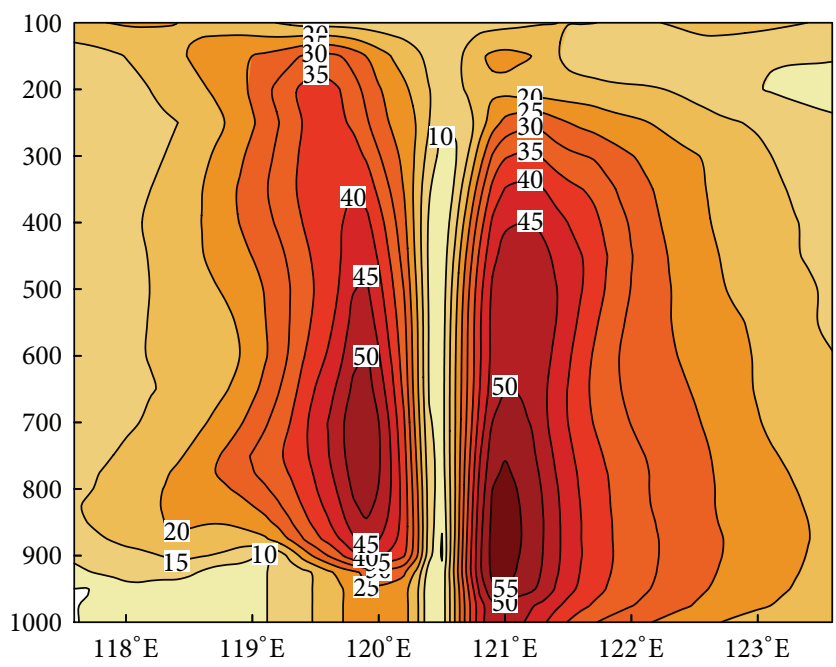

(d) Bopha_str (Saomai at $120.6^{\circ} \mathrm{E}, 27.2^{\circ} \mathrm{N}$ )

Figure 7: Same as Figure 6 except at 1800 UTC 10 August, 2006.

channels. Similar to the analysis in Figure 8, one of them is from southeast flow. The other part of the cluster is drawn back into Bopha through the channels and eventually merges into Bopha. The results verify that the moisture is transported from Saomai to Bopha at the intensifying stage and from Bopha to Saomai at weakening stage through the channel.

As a summary, we portray the relative role of Bopha to the development of Saomai in a schematic diagram (Figure 13). At the beginning, southeast flows are transporting moisture into Saomai. They are divided into two branches after entraining into Saomai: one is ingested into Saomai and becomes part of the Saomai entity; the other one is transported into Bopha, which weakens Saomai in the west side, enhances asymmetry of Saomai, and therefore weakens Saomai. In the absence of Bopha, the moistures would all remain in Saomai and further intensify Saomai as the sensitivity experiments manifest. When they approach close enough, Bopha and
Saomai start rotating cyclonically around the middle point of the connecting line between two TC centers through the BTC interaction process. The rotation changes the relative position in the environment circulation. The directions of moisture transportation through the channels are reversed because of the supply of moisture source. At this stage, Bopha also receives moisture from southeast flows and then also transports moisture into Saomai. Hence, Bopha can intensify Saomai at the later stage.

\section{Conclusion and Discussion}

The relationship between Super Typhoon Saomai and Tropical Storm Bopha is regarded as the BTC interaction. Bopha's effect may play an important role in maintaining and intensifying the intensity of Saomai. We use a mesoscale numerical model particularly designed for the purpose of 


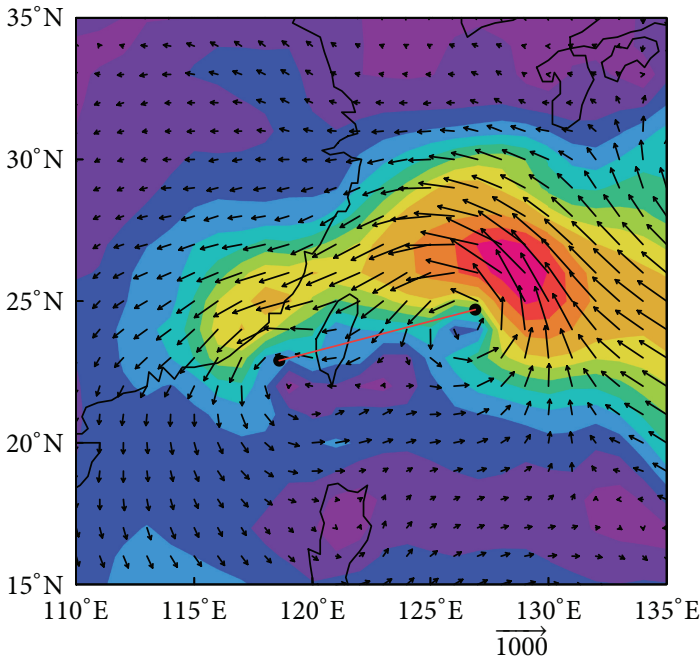

(a) 06Z09AUG2006 column moisture flux

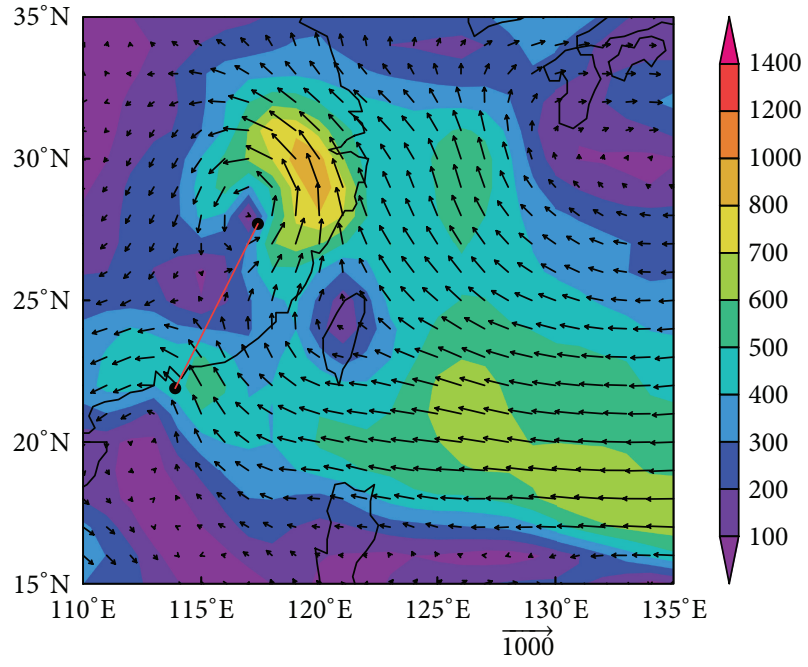

(b) 00Z11AUG2006 column moisture flux

FIGURE 8: Volume moisture fluxes (vectors) with their magnitude (shaded, $\mathrm{kg} \mathrm{m}^{-1} \mathrm{~s}^{-1}$ ) at (a) 0600 UTC 09 August, 2006 , and (b) 0000 UTC 11 August, 2006, computed from the $1^{\circ} \times 1^{\circ}$ NCEP FNL operational global analysis. The red lines connect two centers of Saomai and Bopha.

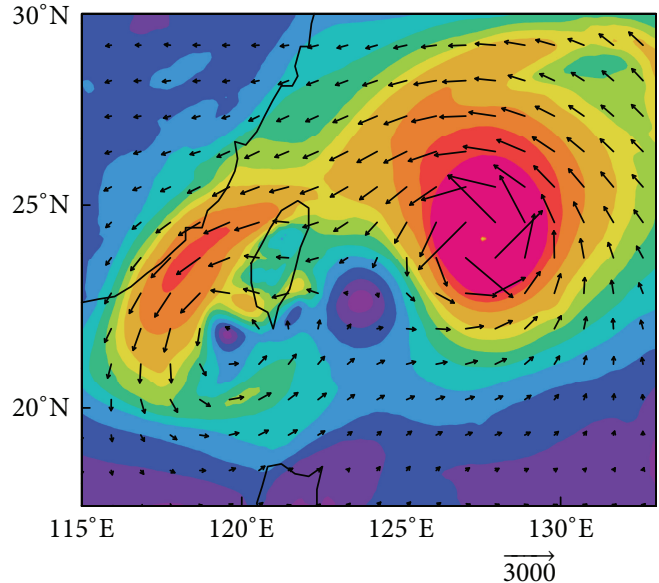

(a)

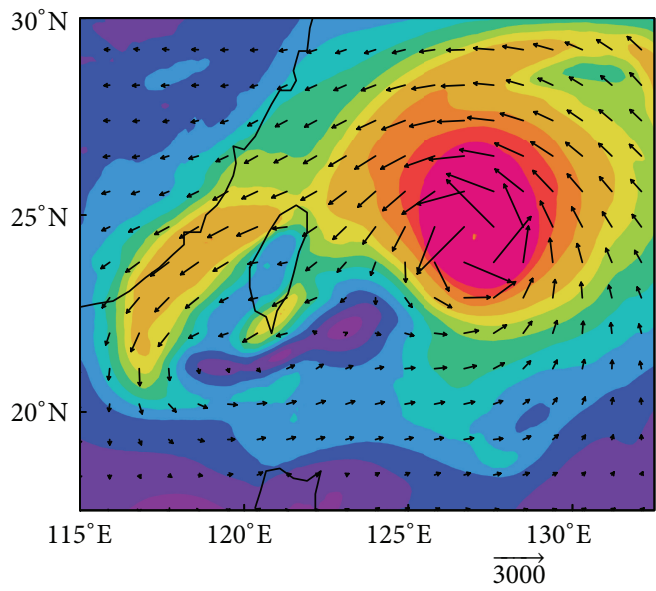

(c)

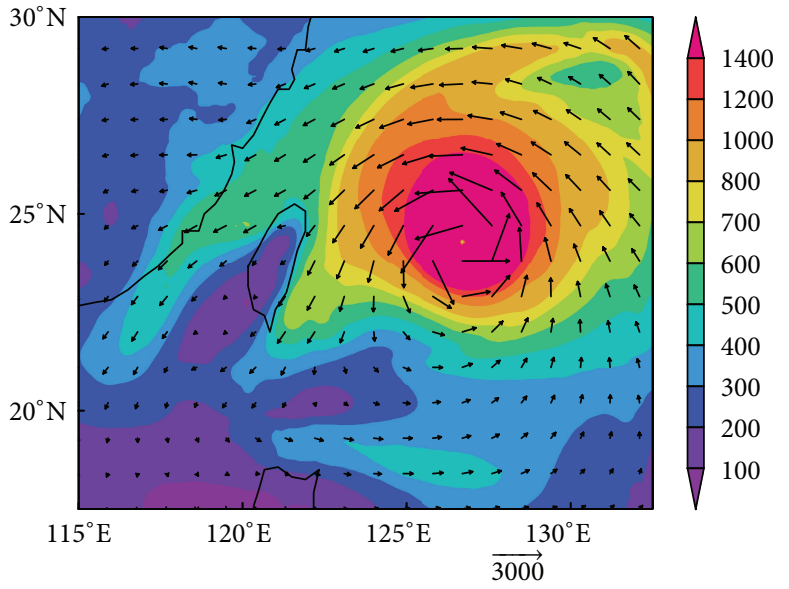

(b)

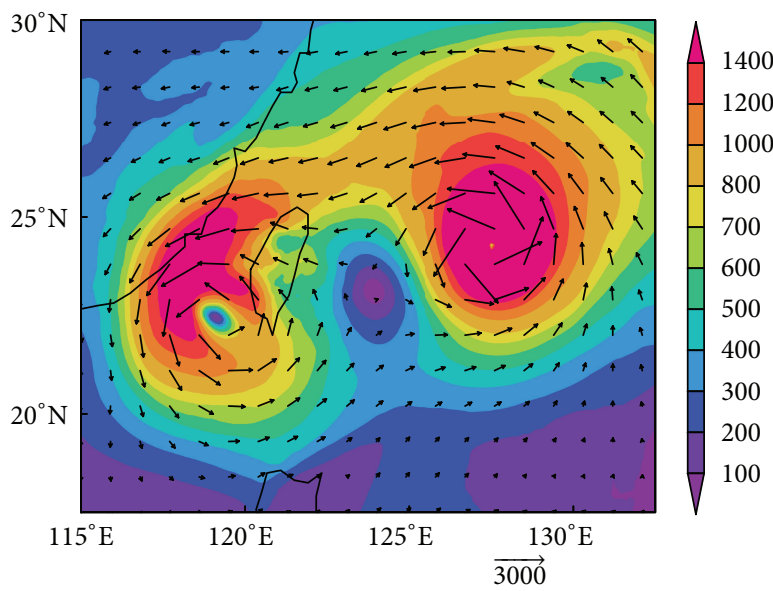

(d)

FIGURE 9: Simulated volume moisture fluxes (vectors) with their magnitude (shaded, $\mathrm{kg} \mathrm{m}^{-1} \mathrm{~s}^{-1}$ ) for CNTR (a), Bopha_rm (b), Bopha_wk (c), and Bopha_str (d) at 0600 UTC 09 August, 2006. 


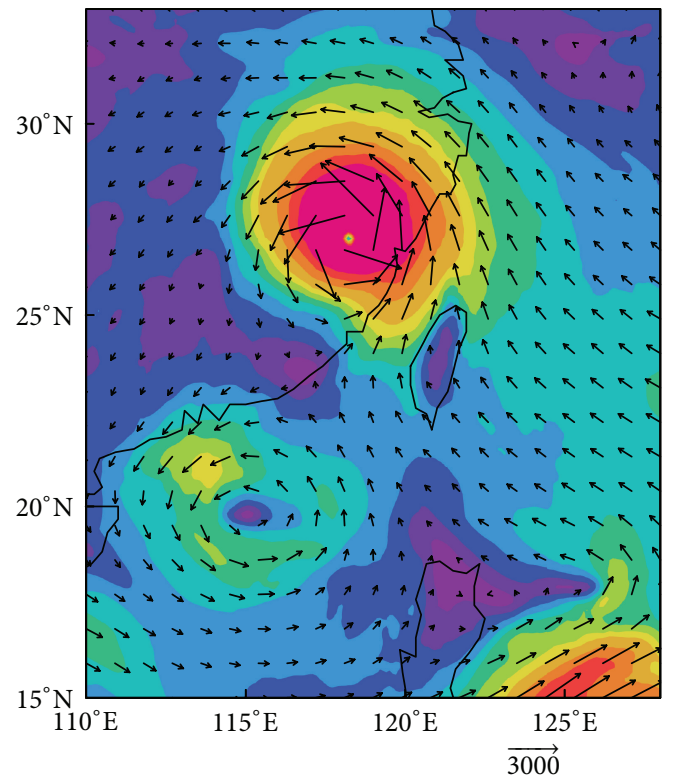

(a)

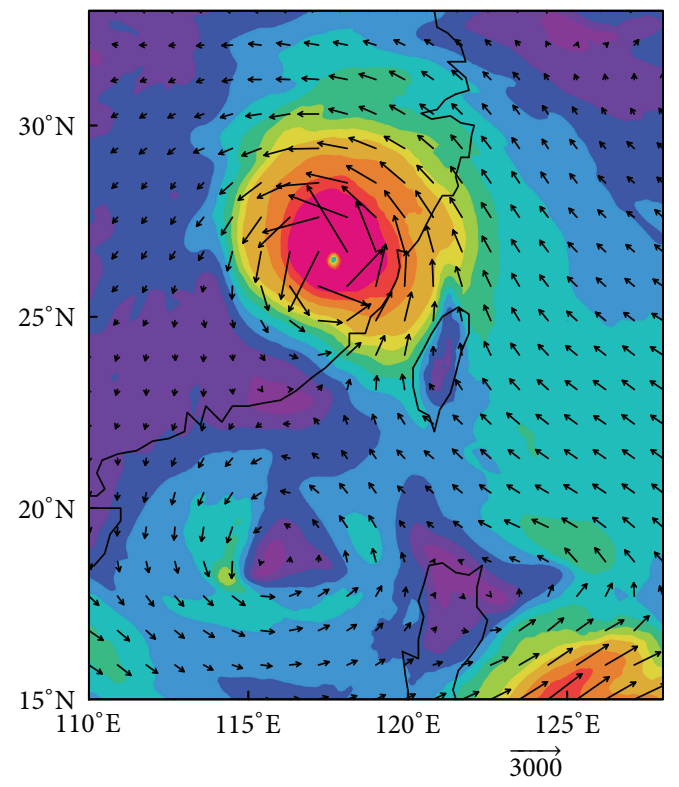

(c)

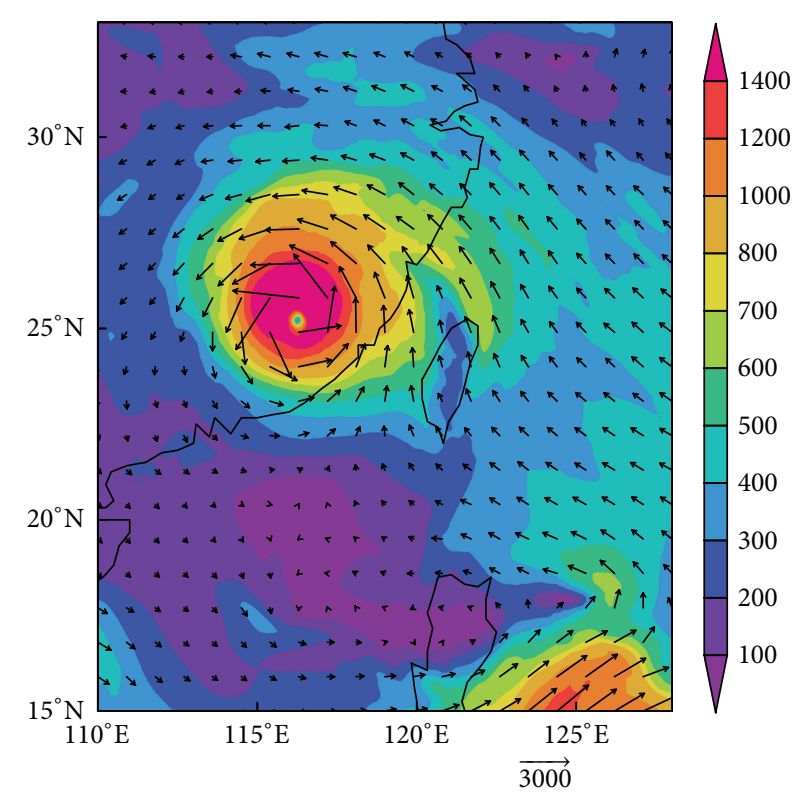

(b)

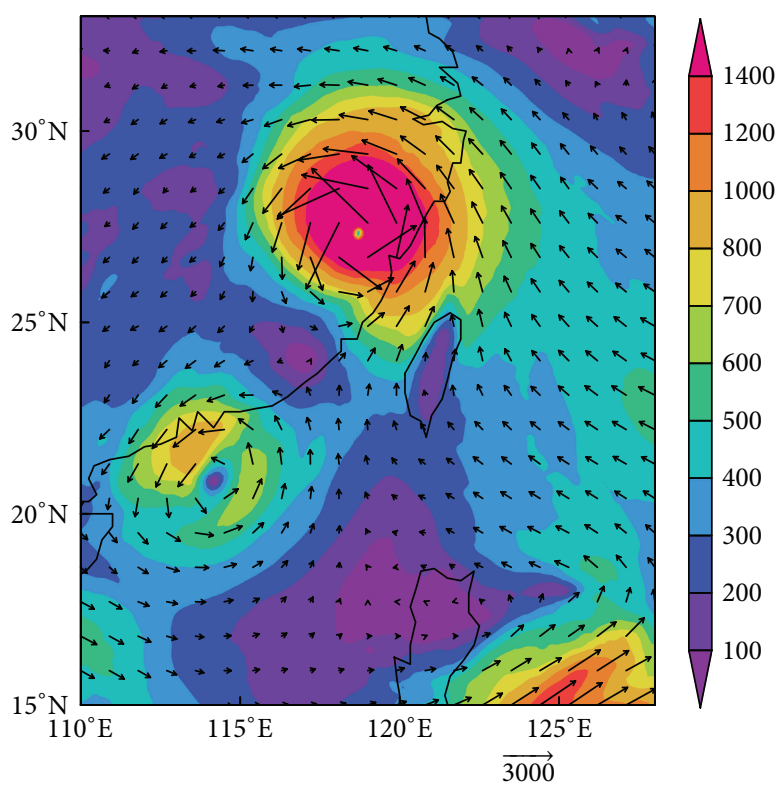

(d)

Figure 10: The same as Figure 9 except at 0000 UTC 11 August, 2006.

hurricane research and forecast, HWRF, to quantify the influence of Bopha on the simulated Saomai. One control and three sensitivity experiments are designed in this study by artificially modifying Bopha intensities in initial conditions through HWRF vortex initialization application.

It is found that CNTR produces the track of Bohpa consistent with the best track estimated by JTWC during the first 30 hours and with a general southward bias in the final $60 \mathrm{~h}$ simulation thereafter. The simulated Saomai track is almost the same as the best track from JTWC expect for slight overestimation of the translation speed after $36 \mathrm{~h}$. Despite some differences in the detailed intensity changes, HWRF simulates reasonably well the intensities during the rapid intensification stage, the sustained stage, and the weakening stage, especially the timing of the intensity change, which is one of the most difficult forecast problems. The detailed intensity verification is not the emphasis in this study because of the deficiency of the best track estimation. The simulation in CNTR further reveals the BTC interaction process. The discrepancy in the best tracks between the JTWC and the CMA arises mainly because of the differences in the way the maximum sustained wind is estimated. The JTWC best track uses 1-minute sustained 10-meter maximum wind while CMA estimates the 2-minute averaged 10-meter wind speed. The more complicated problem is to estimate the sustained or averaged maximum wind over the ocean without surface 


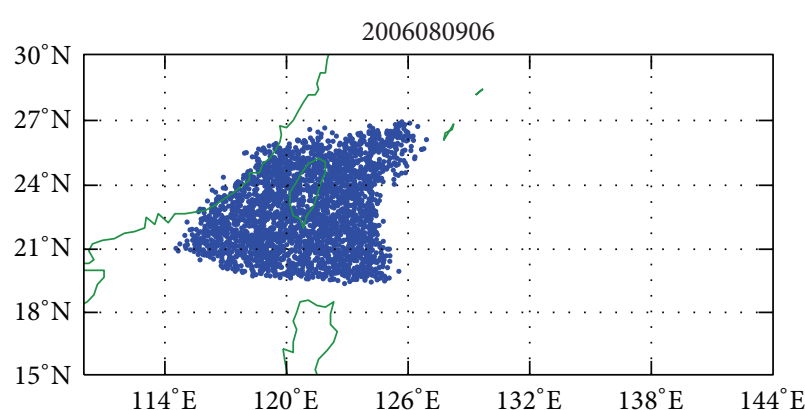

(a)

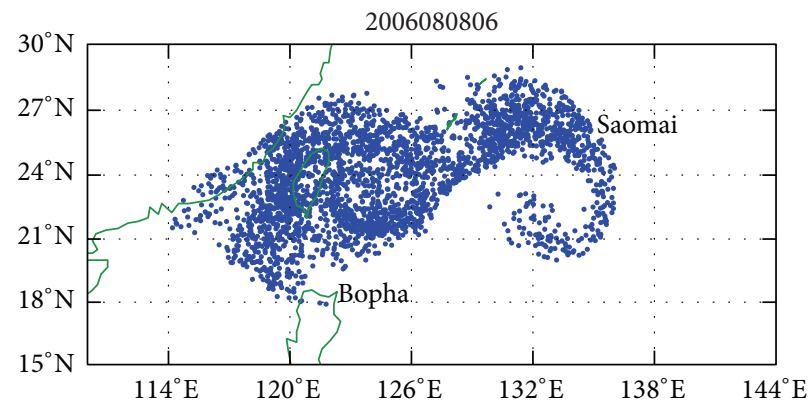

(c)

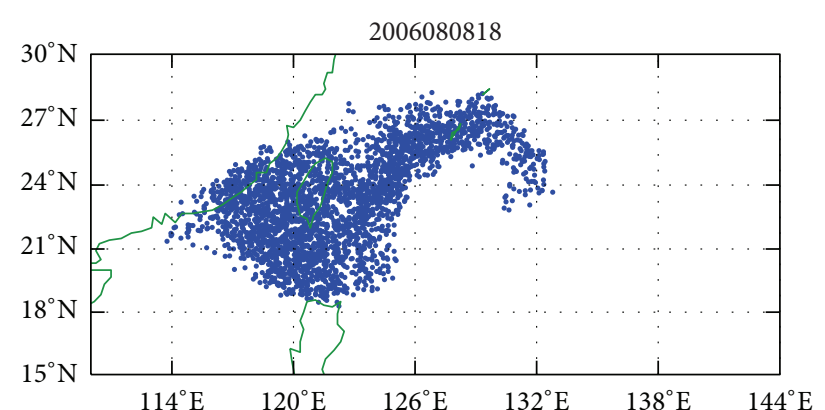

(b)

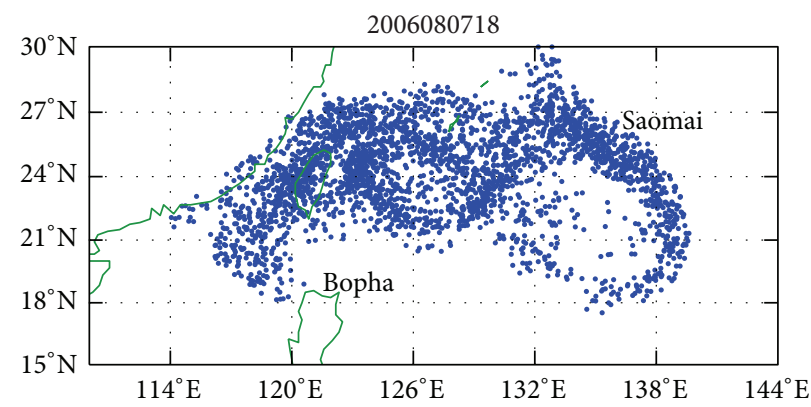

(d)

Figure 11: Back trajectories of the cluster of moisture particles released at 1200 UTC 9 August in Bopha $\left(115.5 \sim 125.5^{\circ} \mathrm{E}, 20.1 \sim 25.0^{\circ} \mathrm{N}\right)$ in the FLEXPART model at (a) 0600 UTC 09 August, 2006, (b) 1800 UTC 08 August, (c) 0600 UTC 08 August, and (d) 1800 UTC 07 August.

observations. This problem is beyond the discussion in this study.

Detailed analyses of the sensitivity experiments show that Bopha has a distinctive effect on Saomai at different stages of its evolution. Saomai is intensified (weakened) and deviates northward when Bopha's initial intensity is increased (weakened) at the weakening stage while the track of Saomai remains unchanged and the intensities are reduced (increased) when Bopha's initial intensity is increased (weakened) at the intensifying stage. It is also shown that the direction change of the moisture transportation within the moisture transport channel, which links Bopha and Saomai, is the key factor in BTC interaction process. This direction change in different stages enforces the opposite role in the intensity evolution of Saomai. In general, increasing Bopha's intensity tends to produce stronger moisture transportation within the channel. More moisture transportation from Saomai to Bopha thus produces more asymmetrical structure and weakens the intensity of Saomai at the intensifying and the reverse course during the weakening stage.

Previous studies have demonstrated that moisture from environment is a key factor for intensification of TCs [28, 29]. Vortex initialization in HWRF is a dynamical adjusting process. All model prognostic variables such as wind, temperature, moisture, and corresponding pressure are rebalanced according to observed TC intensities at the initial time. Therefore, differences of initial conditions can result in different storm-environment/storm-storm interaction. When the distance of Bopha and Saomai was close enough, a moisture transport channel was consequently formed between two TCs. Strength and direction of moisture transport through the channel were modulated by Bopha's initial intensity in these sensitivity tests. Relative location between them can also continuously change when they mutually rotate because of the Fujiwhara effect. Moisture was transported away from Saomai to Bopha at the intensifying stage while the transport direction was reversed at the weakening stage. Noticeably, intensity of Saomai can be enhanced/away by additional moisture transported from/to Bopha overlapping on southeast environmental flow from the ocean. In short, Bopha's intensity can influence the strength of moisture exchange within transport channel and certainly change the moisture budget, resulting in the Saomai's intensity fluctuation. The mutual rotation of Bopha and Saomai can change the relative position of them, therefore, eventually changing the strength and direction of moisture exchange within transport channel, which changes effects on the intensities of Saomai.

We further confirm the previous conclusion by tracing the trajectories of a cluster of water vapor particles based on FLEXPART model. The results demonstrate that the cluster is transported from Saomai to Bopha at the intensifying stage and from Bopha to Saomai at weakening stage. The trajectory analysis also shows the second moisture transport channel for Saomai to maintain its intensity.

Bopha plays an important role on the development of Saomai, especially for maintaining the intensity of Saomai through entraining moisture from Bopha before its landfall. This study manifests that BTC interaction process should be taken into account for forecasting the intensity of TC when one TC approaches another and the critical distance of the $\mathrm{BTC}$ interaction is reached. 


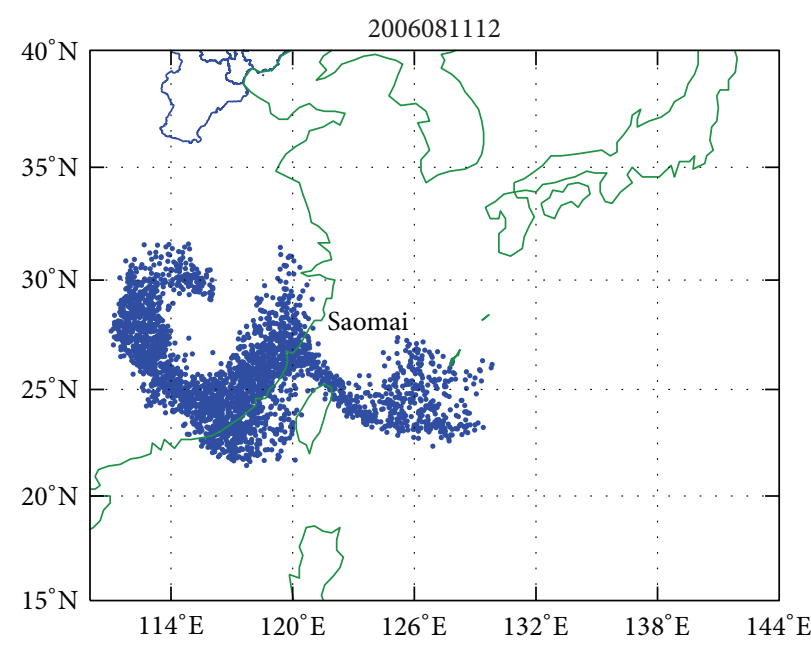

(a)

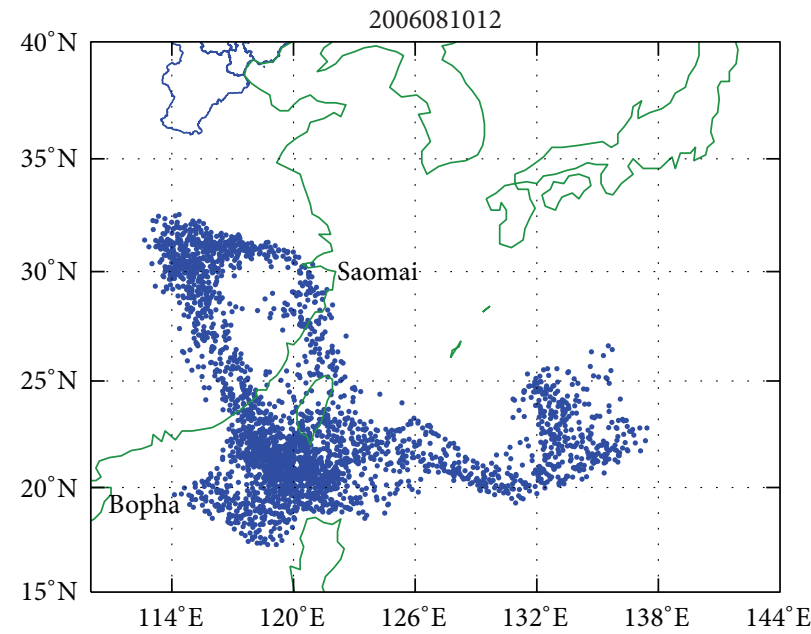

(c)

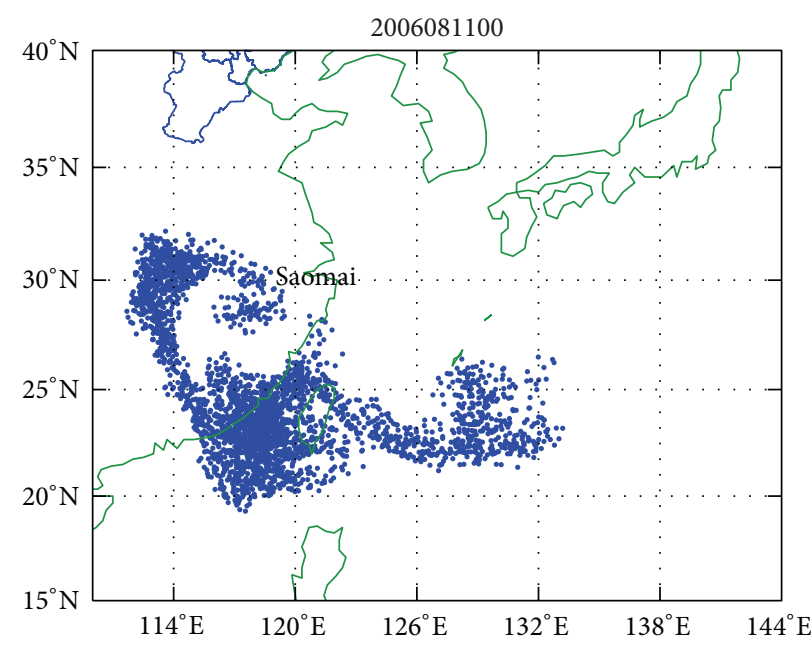

(b)

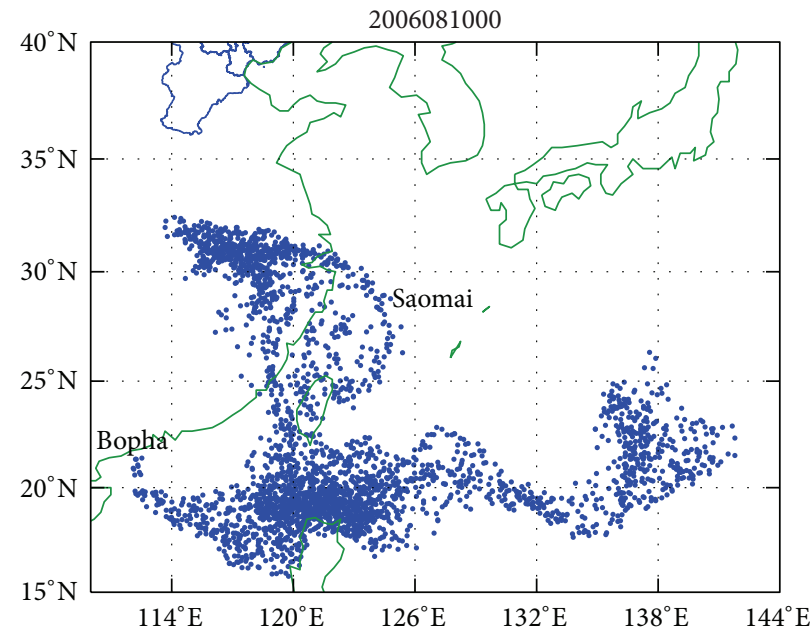

(d)

FIGURE 12: Back trajectories of the cluster of moisture particles released at 0000 UTC 13 August in Saomai $\left(114.5 \sim 120.5^{\circ} \mathrm{E}, 26 \sim 33.5^{\circ} \mathrm{N}\right)$ in the FLEXPART model at (a) 1200 UTC 11 August, (b) 0000 UTC 11 August, (c) 1200 UTC 10 August, and (d) 0000 UTC 10 August, 2006.

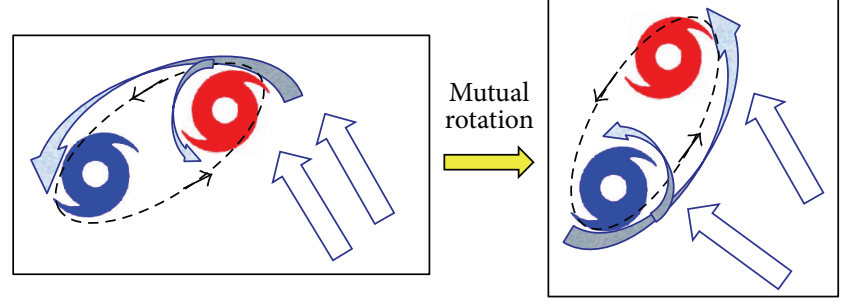

FIGURE 13: Schematic diagram portraying the TC mutual rotation and binary TC interaction process. The thick arrows donate the moisture flux source; the solid blue arrows donate the moisture flux channel; the dashed arrows illustrate the cyclonic rotation.

\section{Acknowledgments}

Hongxiong $\mathrm{Xu}$ is a current Ph.D. degree student of Nanjing University of Information Science and Technology. Hongxiong $\mathrm{Xu}$ and Xiangde Xu's research was jointly supported by the China National Key Basic Research and Development Projects (973 Projects) under the Grant 2009CB421504 and the National Natural Science Foundation of China (General Program) under the Grant 41075037. Xuejin Zhang was funded by NOAA's Hurricane Forecast Improvement Program (Grant no. NA12NWS4680007). Authors acknowledge valuable comments by two anonymous reviewers and Dr. Sim Aberson, Robert Black, and Dr. Hua Chen from AOML/HRD. HWRF system was downloaded from Developmental Testbed Center (DTC), which is sponsored by NOAA, AFWA, and NSF.

\section{References}

[1] F. D. Marks, L. K. Shay, G. Barnes et al., "Landfalling tropical cyclones: forecast problems and associated research opportunities," Bulletin of the American Meteorological Society, vol. 79, no. 2, pp. 305-323, 1998. 
[2] B. W. Shen, W. K. Tao, W. K. Lau, and R. Atlas, "Predicting tropical cyclogenesis with a global mesoscale model: Hierarchical multiscale interactions during the formation of tropical cyclone Nargis (2008)," Journal of Geophysical Research D, vol. 115, no. 14, Article ID D14102, 2010.

[3] S. Fujiwhara, "The mutual tendency towards symmetry of motion and its application as a principle in meteorology," Quarterly Journal of the Royal Meteorological Society, vol. 47, pp. 287-293, 1921.

[4] S. Fujiwhara, "The mutual tendency towards symmetry of motion and its application as a principle in meteorology," Quarterly Journal of the Royal Meteorological Society, vol. 49, pp. 287-293, 1923.

[5] S. Fujiwhara, "On the growth and decay of vortical systems," Quarterly Journal of the Royal Meteorological Society, vol. 49, pp. 75-104, 1931.

[6] S. Brand, "Interaction of binary tropical cyclones of the Western North pacific ocean," Journal of Applied Meteorology, vol. 9, no. 3, pp. 433-441, 1970.

[7] L. E. Carr, M. A. Boothe, and R. L. Elsberry, "Observational evidence for alternate modes of track-altering binary tropical cyclone scenarios," Monthly Weather Review, vol. 125, no. 9, pp. 2094-2111, 1997.

[8] L. E. Carr and R. L. Elsberry, "Objective diagnosis of binary tropical cyclone interactions for the western north pacific basin," Monthly Weather Review, vol. 126, no. 6, pp. 1734-1740, 1998.

[9] L. Chen and Z. Meng, "An overview on tropical cyclone research progress in china during the past ten years," Chinese Journal of Atmospheric Sciences, vol. 5, no. 3, pp. 420-432, 2001.

[10] L. Chen, X. Xu, Z. Luo, and J. Wang, Introduction to Tropical Cyclone Dynamics, Meteorological Press, Beijing, China, 2002.

[11] D. Wei and J. Zhang, "A fluid dynamic simulation study on binary typhoon interaction," Science in China, vol. 12, no. 1, pp. 87-94, 1982.

[12] H. C. Kuo, G. T. J. Chen, and C. H. Lin, "Merger of tropical cyclones Zeb and Alex," Monthly Weather Review, vol. 128, no. 8, pp. 2967-2975, 2000.

[13] C. C. Yang, C. C. Wu, K. H. Chou, and C. Y. Lee, "Binary interaction between Typhoons Fengshen (2002) and Fungwong (2002) based on the potential vorticity diagnosis," Monthly Weather Review, vol. 136, no. 12, pp. 4593-4611, 2008.

[14] X. Xu, C. Lu, H. Xu, and L. Chen, "A possible mechanism responsible for exceptional rainfall over Taiwan from Typhoon Morakot," Atmospheric Science Letters, vol. 12, no. 3, pp. 294299, 2011.

[15] X. Wu, J. Fei, X. Huang, X. Zhang, X. Cheng, and J. Ren, "A numerical study of the interaction between two simultaneous storms: goni and morakot in september 2009," Advances in Atmospheric Sciences, vol. 29, no. 3, pp. 561-574, 2012.

[16] S. Gopalakrishnan, Q. Liu, T. Marchok et al., Hurricane Weather Research and Forecasting (HWRF) Model Scientific Documentation, 2010.

[17] S. G. Gopalakrishnan, F. Marks, X. Zhang, J. W. Bao, K. S. Yeh, and R. Atlas, "The experimental HWRF system: a study on the influence of horizontal resolution on the structure and intensity changes in tropical cyclones using an idealized framework," Monthly Weather Review, vol. 139, no. 6, pp. 1762-1784, 2011.

[18] W. C. Skamarock, J. B. Klemp, J. Dudhia et al., "A description of the advanced research WRF version 3," NCAR TECHNICAL NOTE NCAR/TN-475+STR, 2008.
[19] Z. I. Janjic, J. P. Gerrity, and S. Nickovic, "An alternative approach to nonhydrostatic modeling," Monthly Weather Review, vol. 129, no. 5, pp. 1164-1178, 2001.

[20] Z. I. Janjic, "A nonhydrostatic model based on a new approach," Meteorology and Atmospheric Physics, vol. 82, no. 1-4, pp. 271285, 2003.

[21] K. S. Yeh, X. Zhang, S. Gopalakrishnan et al., "Performance of the experimental HWRF in the 2008 hurricane season," Natural Hazards, vol. 63, no. 3, pp. 1439-1449, 2012.

[22] Y. Kurihara, M. A. Bender, R. E. Tuleya, and R. J. Ross, "Improvements in the GFDL hurricane prediction system," Monthly Weather Review, vol. 123, no. 9, pp. 2791-2801, 1995.

[23] S. Saha, S. Moorthi, H. L. Pan et al., "NCEP Climate Forecast System Reanalysis (CFSR) 6-hourly Products, January 1979 to December 2010," Research Data, The National Center for Atmospheric Research, Computational and Information Systems Laboratory, Boulder, Colo, USA, 2010.

[24] A. Stohl, C. Forster, A. Frank, P. Seibert, and G. Wotawa, “Technical note: the lagrangian particle dispersion model FLEXPART version 6.2," Atmospheric Chemistry and Physics, vol. 5, no. 9, pp. 2461-2474, 2005.

[25] S. Eckhardt, A. J. Prata, P. Seibert, K. Stebel, and A. Stohl, "Estimation of the vertical profile of sulfur dioxide injection into the atmosphere by a volcanic eruption using satellite column measurements and inverse transport modeling," Atmospheric Chemistry and Physics, vol. 8, no. 14, pp. 3881-3897, 2008.

[26] K. A. Emanuel and M. Živković-Rothman, "Development and evaluation of a convection scheme for use in climate models," Journal of the Atmospheric Sciences, vol. 56, no. 11, pp. 1766-1782, 1999.

[27] C. Forster, U. Wandinger, G. Wotawa et al., "Transport of boreal forest fire emissions from Canada to Europe," Journal of Geophysical Research D, vol. 106, no. 19, pp. 22887-22906, 2001.

[28] G. J. Holland, "The maximum potential intensity of tropical cyclones," Journal of the Atmospheric Sciences, vol. 54, no. 21, pp. 2519-2541, 1997.

[29] K. A. Emanuel, “The maximum intensity of hurricanes," Journal of the Atmospheric Sciences, vol. 45, no. 7, pp. 1143-1155, 1988. 

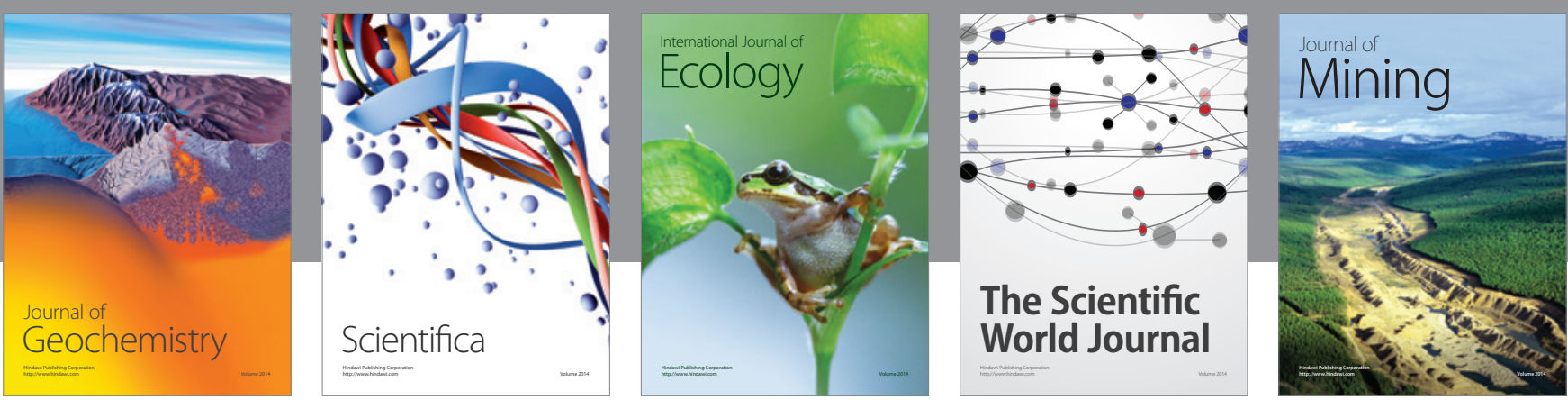

The Scientific World Journal
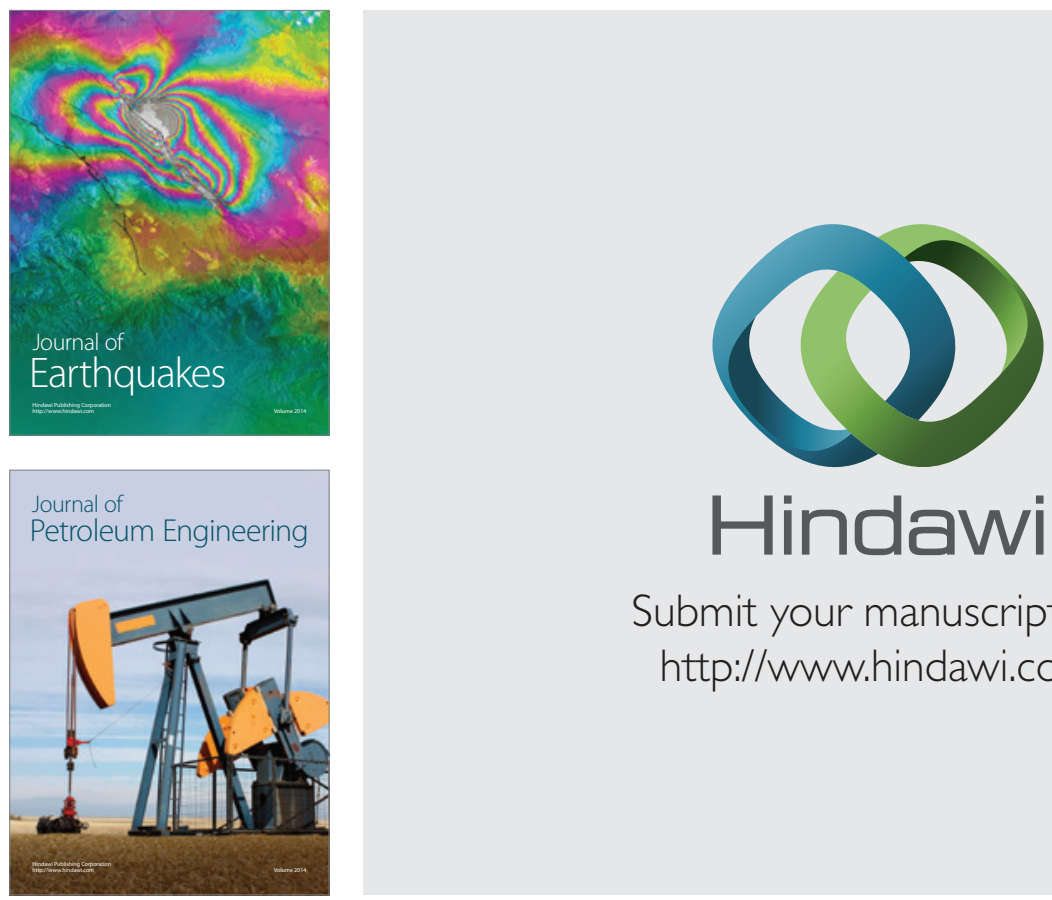

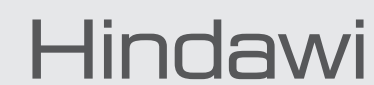

Submit your manuscripts at

http://www.hindawi.com
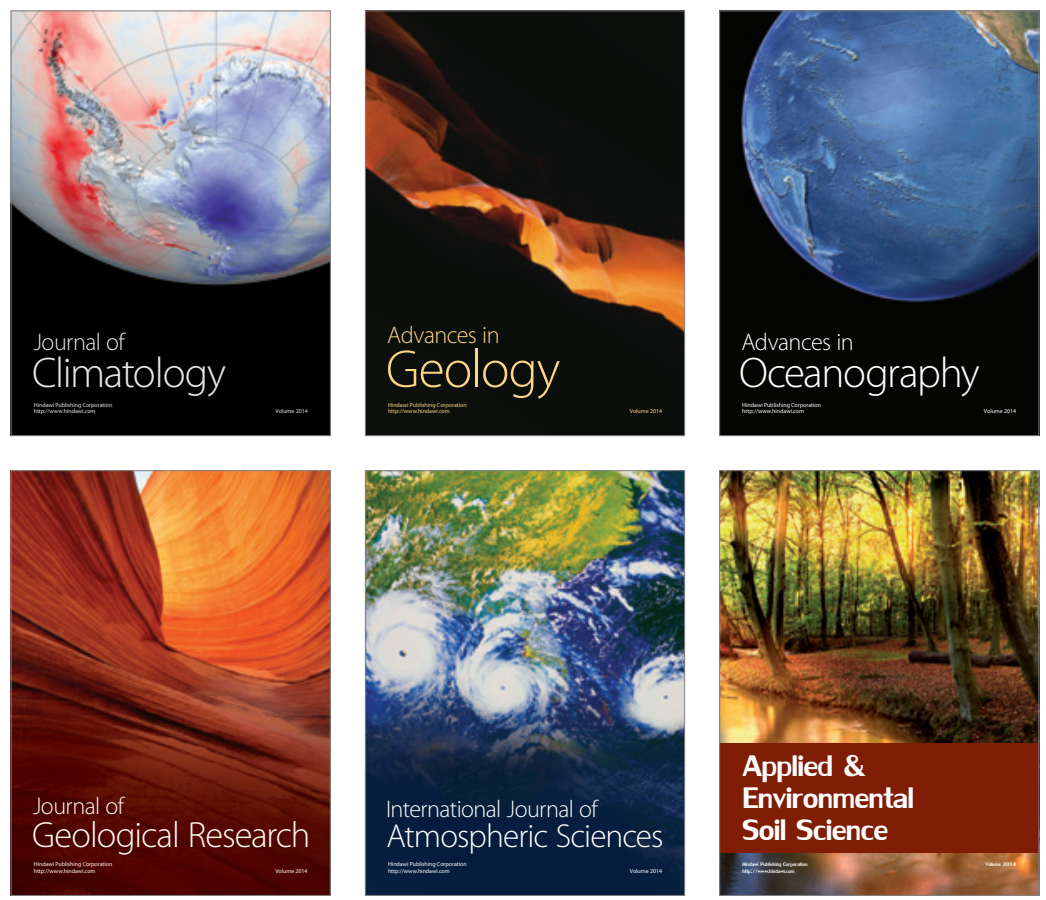
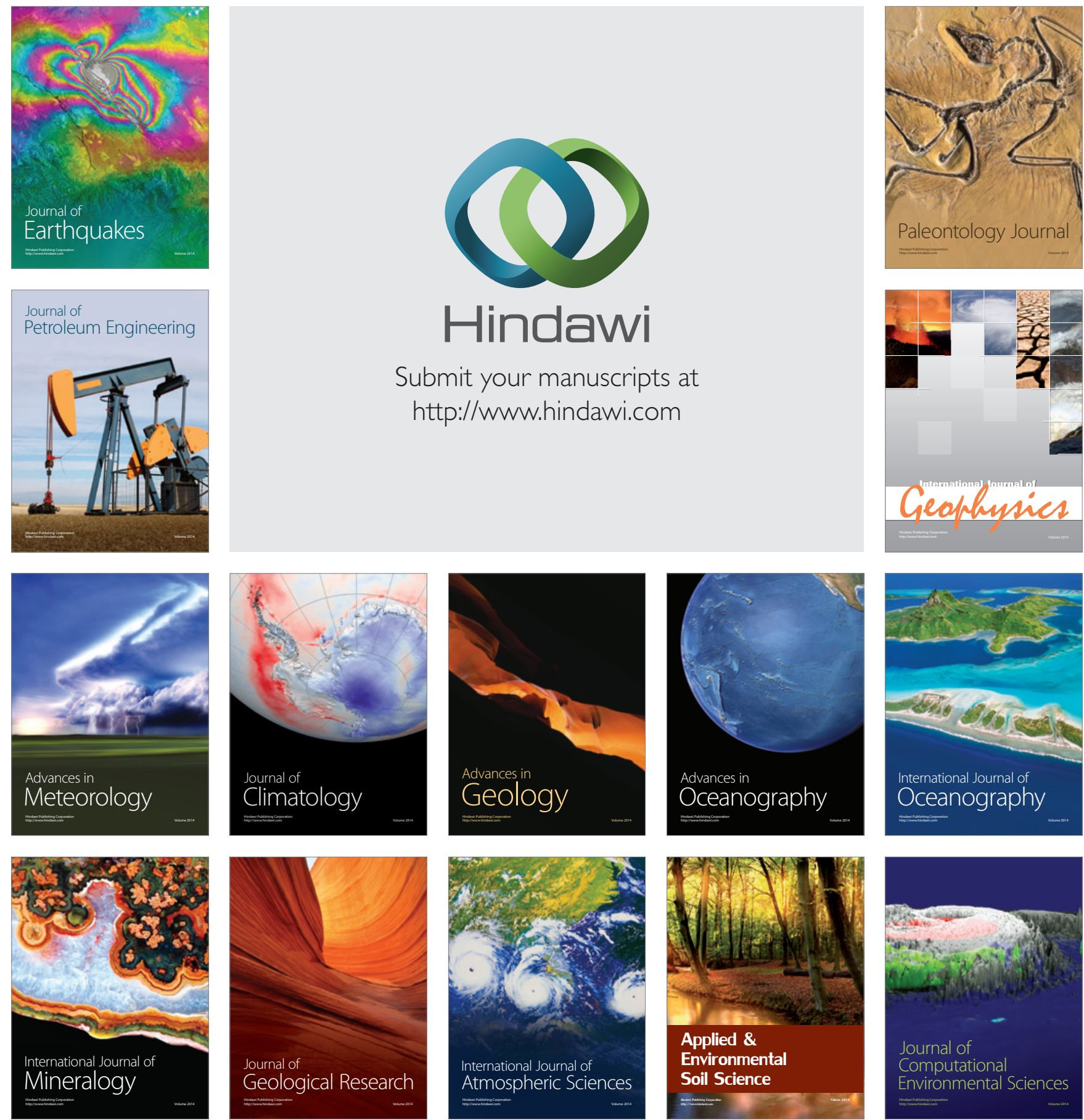